\title{
Age-specific microbiota in altering host inflammatory and metabolic signaling as well as metabolome based on the sex
}

\author{
Lili Sheng ${ }^{1,2 \#}$, Prasant Kumar Jena ${ }^{1,3 \#}$, Ying Hu ${ }^{1}$, Yu-Jui Yvonne Wan ${ }^{1}$ \\ ${ }^{1}$ Department of Pathology and Laboratory Medicine, Davis Health, University of California, Sacramento, CA, USA; ${ }^{2}$ Institute of Interdisciplinary \\ Integrative Medicine Research, Shanghai University of Traditional Chinese Medicine, Shanghai, China; ${ }^{3}$ Department of Pediatrics, Cedars Sinai \\ Medical Center, Los Angeles, CA, USA \\ Contributions: (I) Conception and design: YJY Wan; (II) Administrative support: None; (III) Provision of study materials or patients: None; (IV) \\ Collection and assembly of data: L Sheng, PK Jena, Y Hu; (V) Data analysis and interpretation: All authors; (VI) Manuscript writing: All authors; (VII) \\ Final approval of manuscript: All authors. \\ \#These authors contributed equally to this work. \\ Correspondence to: Yu-Jui Yvonne Wan, PhD. Department of Pathology and Laboratory Medicine, Davis Health, University of California, Sacramento \\ 95817, CA, USA. Email: yjywan@ucdavis.edu.
}

Background: Metabolism is sex-different, and the direct link between gut microbiota and aging-associated metabolic changes needs to be established in both sexes.

Methods: Gene expression, metabolic and inflammatory signaling, gut microbiota profile, and metabolome were studied during aging and after fecal microbiota transplantation (FMT) in mice of both sexes.

Results: Our data revealed young female mice and aged male mice were the most insulin sensitive and resistant group, respectively. In addition, aging reduced sex difference in insulin sensitivity. Such ageand sex-dependent metabolic phenotypes were accompanied by shifted gut microbiota profile and altered abundance of bacterial genes that produce butyrate, propionate, and bile acids. After receiving feces from the aged males (AFMT), the most insulin-resistant group, recipients of both sexes had increased hepatic inflammation and serum endotoxin. However, AFMT only increased insulin resistance in female mice and abolished sex difference in insulin sensitivity. Additionally, such changes were accompanied by narrowed sex difference in metabolome. Metabolomics data revealed that age-associated insulin resistance in males was accompanied by increased sugar alcohols and dicarboxylic acids as well as reduced aromatic and branchedchain amino acids. Further, receiving feces from the young females (YFMT), the most insulin-sensitive group, reduced body weight and fasting blood glucose in male recipients and improved insulin sensitivity in females, leading to enhanced sex differences in insulin sensitivity and metabolome.

Conclusions: Aging systemically affected inflammatory and metabolic signaling based on the sex. Gut microbiome is age and sex-specific, which affects inflammation and metabolism in a sex-dependent manner.

Keywords: Aging; microbiome; metabolome; insulin sensitivity; fecal transplantation

Submitted Aug 19, 2020. Accepted for publication Nov 25, 2020.

doi: 10.21037/hbsn-20-671

View this article at: http://dx.doi.org/10.21037/hbsn-20-671

\section{Introduction}

Altered gut microbial ecosystems have been associated with increased risk of metabolic and immune disorders $(1,2)$. Aging is associated with chronic inflammation, a risk for age-associated pathologies such as atherosclerosis, insulin resistance, diabetes, as well as Alzheimer's disease $(3,4)$. Emerging evidence reveals aging-associated changes in the composition, diversity, and function of gut microbiota increases gut permeability and activates innate immune responses $(5,6)$. Therefore, microbiome-based interventions against aging-associated health issues should provoke 
attention.

The microbiota-targeted interventions slow down aging process through preventing insulin resistance, improving immunity, suppressing chronic inflammation, as well as regulating metabolism (7-9). Additionally, fecal microbiota transplantation (FMT) extends mouse lifespan (10). Moreover, donor metabolic characteristics drive the effects of FMT on recipient insulin sensitivity in male adult (11). Furthermore, feces from lean donors can transiently improve the insulin sensitivity in some obese male patients with metabolic syndrome, and the improvement is driven by baseline intestinal microbiota composition of the recipients $(12,13)$. These findings suggested the importance of donor as well as recipient in dictating the transplantation outcomes. Additional studies are needed to understand the sex effect.

Women and men differ substantially regarding the degree of insulin sensitivity, body composition, energy balance, and the incidence of metabolic diseases (14). Others' and our studies show sex differences in microbiota may account for sex dissimilarity in metabolism and metabolic diseases (15-17). However, whether the sex of donor and recipient affect FMT efficacy in metabolism has not been examined.

In the current study, we tested a hypothesis that agingassociated metabolic issues such as insulin resistance may be due to aging-induced structural and functional changes of the gut microbiome in a sex-dependent manner. Thus, we analyzed aging-associated gut microbiota and metabolome on inflammatory signaling and metabolism in both sexes. Our novel data revealed that aging differentially affects metabolic signaling and metabolome in males and females. Additionally, sex difference in insulin sensitivity narrowed as mice age. Because aged male mice were the most insulin resistant group, whereas young female mice were the most insulin sensitive group, FMT were performed by using aged male feces (AFMT) and young female feces (YFMT). Our data showed that AFMT lead to insulin resistance only in females, which abolished sex difference in insulin sensitivity and colon metabolome. Moreover, YFMT reduced body weight and fasting blood glucose in males and improved insulin sensitivity in females, leading to increased sex differences in insulin sensitivity and colon metabolome. Together, FMT effects on metabolic changes are sex specific.

We present the following article in accordance with the ARRIVE reporting checklist (available at https://hbsn. amegroups.com/article/view/10.21037/hbsn-20-671/rc).

\section{Methods}

\section{Animal studies}

Specific pathogen-free male and female C57BL/6 wild-type (WT) mice (Jackson Laboratory, Sacramento, CA, USA) were fed with a normal diet (5\% fat, $12 \%$ sucrose, and $0.01 \%$ cholesterol, w/w, Harlan Teklad, Indianapolis, IN, USA) after weaning ( 3 weeks). Mice were euthanized at 5, 10 , and 15 months of age $(n=8)$. For aged FMT (AFMT), aged feces were collected from 20-month-old WT male mice. As suggested by recent publication (18), fresh feces obtained from four mice were pooled in sterile phosphatebuffered saline (PBS) with 25\% glycerol and stored at $-80{ }^{\circ} \mathrm{C}$ for further FMT. For AFMT, recipients were 3 -week-old mice of both sexes, and they received PBS (control group) or pooled aged fecal material $30 \mathrm{mg}$ feces $/ 200 \mu \mathrm{L}$, AFMT group) by oral gavage twice per week for 4 months. Mice were euthanized when they were 5 months of age $(n=4)$. For young FMT (YFMT), fresh feces were obtained from 2-month-old WT female mice, pooled in PBS with $25 \%$ glycerol, and stored at $-80{ }^{\circ} \mathrm{C}$ for further FMT. For YFMT, 10-month-old mice of both sexes received PBS (control group) or pooled fecal material (YFMT group) by oral gavage daily for 1.5 months, and mice were euthanized at the age of 11.5 months. Samples were collected and immediately frozen at $-80{ }^{\circ} \mathrm{C}$ for further analysis. Experiments were conducted in accordance with the National Institutes of Health Guide for the Care and Use of Laboratory Animals under protocols (No. 18274) approved by the Institutional Animal Care and Use Committee of the University of California, Davis.

\section{Gene expression profiling}

RNA was extracted from liver, ileum, and visceral fat using TRIzol Reagent (Invitrogen, Carlsbad, CA, USA), and cDNA was synthesized using High Capacity RNA-tocDNA Kit (Applied Biosystems, Foster City, CA, USA). qRT-PCR was performed on an ABI 7900HT Fast realtime PCR system using Power SYBR ${ }^{\circledR}$ Green PCR Master Mix (Applied Biosystems). Primers were designed using Primer3 Input Software (v0.4.0) and the sequences are listed in Table S1. The mRNA levels were normalized to the level of Gapdh mRNA.

\section{Biochemical analysis and insulin tolerance test (ITT)}

Serum lipopolysaccharides (LPS) (Thermo Fisher Scientific, 
Waltham, MA, USA) and alanine transaminase (ALT) (Pointe Scientific, Canton, MI, USA) levels were quantified according to the manufacturer's instructions. After the mice were unfed for $6 \mathrm{~h}$, tail vein blood was used to establish fasting blood glucose levels. For ITT, insulin (1 or $0.75 \mathrm{U}$ per kilogram body weight, i.p.; Millipore Sigma, Burlington, MA, USA) was given followed by monitoring blood glucose levels at various times with the OneTouch Ultra 2 (Johnson \& Johnson Co., New Brunswick, NJ, USA). The area under the curve (AUC) of the blood glucose levels over time was calculated.

\section{Untargeted metabolomic study}

Colon content was extracted, and metabolite levels were quantified by gas chromatography time-of-flight mass spectrometry (GC-TOFMS) at West Coast Metabolomics Center, University of California, Davis (19). Acquired spectra were processed using the BinBase database, filtered, and matched against the Fiehn Mass Spectral Library of 1,200 authentic metabolite spectra with retention index and mass spectrum information or against the National Institute of Standards and Technology (NIST) library. Partial least squares-discriminant analysis and pathway analysis were generated by MetaboAnalyst 3.0. Chemical similarity enrichment analysis was performed by ChemRICH (20).

\section{Quantification of protein}

Hepatic protein lysates $(40 \mathrm{mg})$ were subjected to polyacrylamide gel electrophoresis under reducing conditions following by transferring to polyvinylidene difluoride membranes. The membranes were incubated with $5 \%$ nonfat milk followed by antibody specific for TNF- $\alpha$ (Bioss Antibodies, Woburn, MA, USA), IL-6 (Bioss Antibodies), and $\beta$-actin (Cell Signaling Technology, Danvers, MA, USA). Membranes were then incubated with horseradish peroxidase conjugated secondary antibodies. The signals were detected using an ECL enhanced chemiluminescence system with Pierce SuperSignal West Pico chemiluminescent substrates (Thermo Fisher Scientific).

\section{Quantification of bacterial DNA and $16 S$ rRNA gene sequencing}

Fecal (0.1 gram) DNA was extracted using ZR Fecal DNA MiniPrep Kit (Zymo Research, Irvine, CA, USA), quantified by NanoDrop (Thermo Fisher Scientific), and amplified using primers based on published sequences (Table S1). A dissociation step was included to analyze the melting profile of amplified products. In parallel, qPCR was done using 10-fold serial diluted synthetic DNA fragments of bacterial gene with known concentrations. Bacterial DNA concentration was calculated using standard curves of diluted synthetic DNA fragment. Sequencing of tagged 16S rRNA gene amplicons of fecal DNA was done based on published methods $(21,22)$. The $\mathrm{V} 4$ region of $16 \mathrm{~S}$ rRNA gene was amplified and sequenced using Illumina MiSeq.

\section{Bioinformatics and statistical analysis}

Sequence reads were analyzed according to previous publications $(15,22,23)$. Spearman's correlations were performed with $\mathrm{R}$ statistical software. Data are expressed as mean \pm SD. Differences between groups in microbiota family level were calculated by Kruskal-Wallis test. All other comparisons were calculated by two-tailed Student's $t$-test, one-way ANOVA, or two-way ANOVA followed by Tukey's test using GraphPad Prism 6 software. P values are adjusted for multiple comparisons using false discovery rate. $\mathrm{P}<0.05$ was considered statistically significant.

\section{Results}

\section{Aging differentially affects inflammatory and metabolic signaling}

Many age-related diseases share an inflammatory pathogenesis, the expression of inflammatory genes was studied in the liver and the visceral fat of both sexes at 5, 10, and 15 months of age. Aging increased the expression of genes implicated in inflammatory signaling. For example, aging increased the expression of Tnfa (tumor necrosis factor alpha), Il6 (interleukin 6), Il1 $\beta$ (interleukin 1 beta), Saa1 (serum amyloid A1), Icam1 (intercellular adhesion molecule 1), and Mcp1 (monocyte chemoattractant protein 1 ) in the liver of female mice, while increased Tnfa, Il6, and $M c p 1$ in male mice (Figure 1A,B). Similarly, Tnfa, Ifn $\gamma$ (interferon gamma), and $M c p 1$ in the visceral fat were all elevated during aging in both sexes (Figure 1C).

In contrast to inflammatory signaling, as mice aged, the expression of metabolic genes had the tendency to reduce. Examples are gluconeogenic genes Pepck (phosphoenolpyruvate carboxykinase) and G6pase (glucose6-phosphatase) in the liver and the visceral fat (Figure 1D,E). 

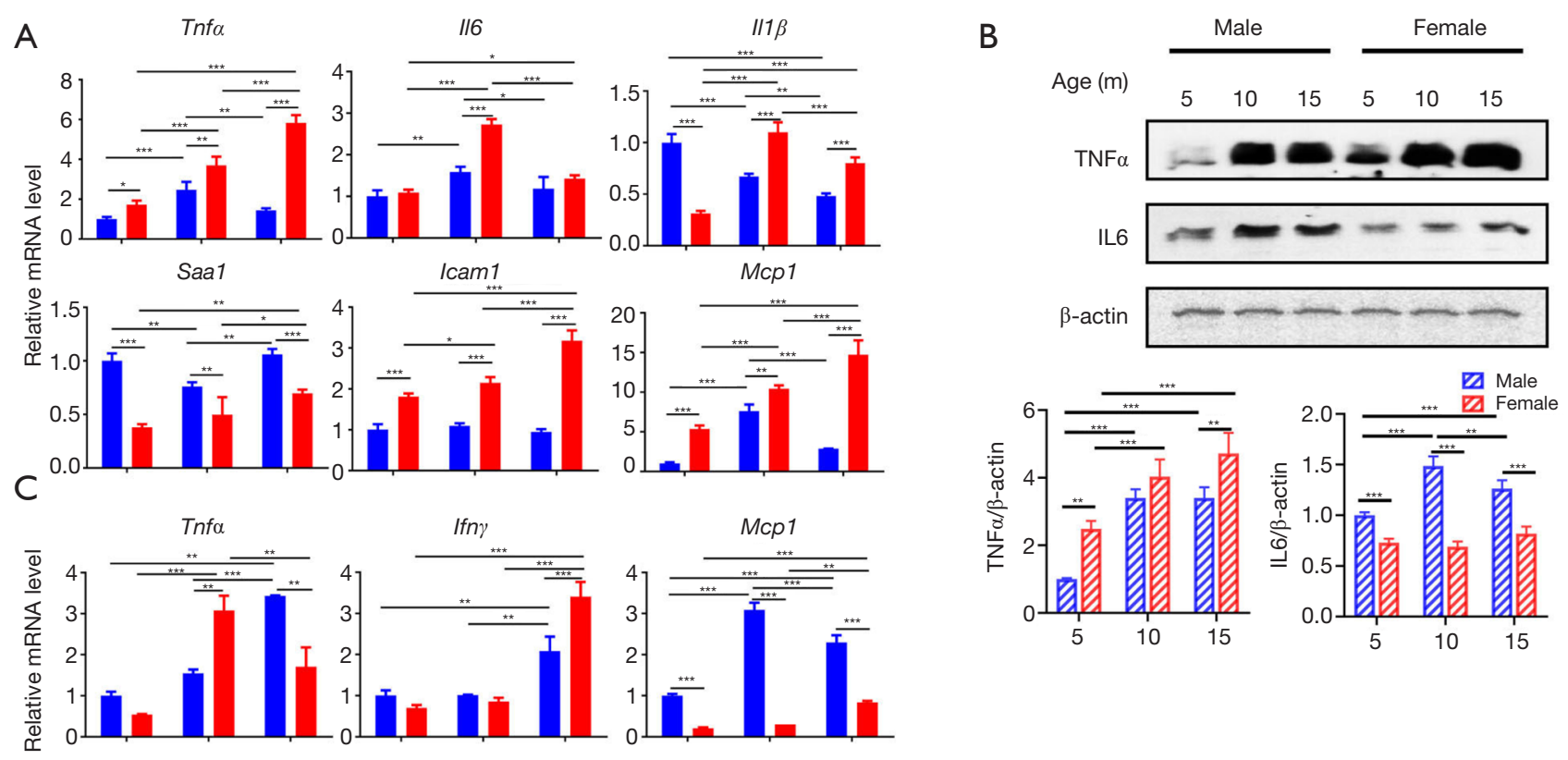

D
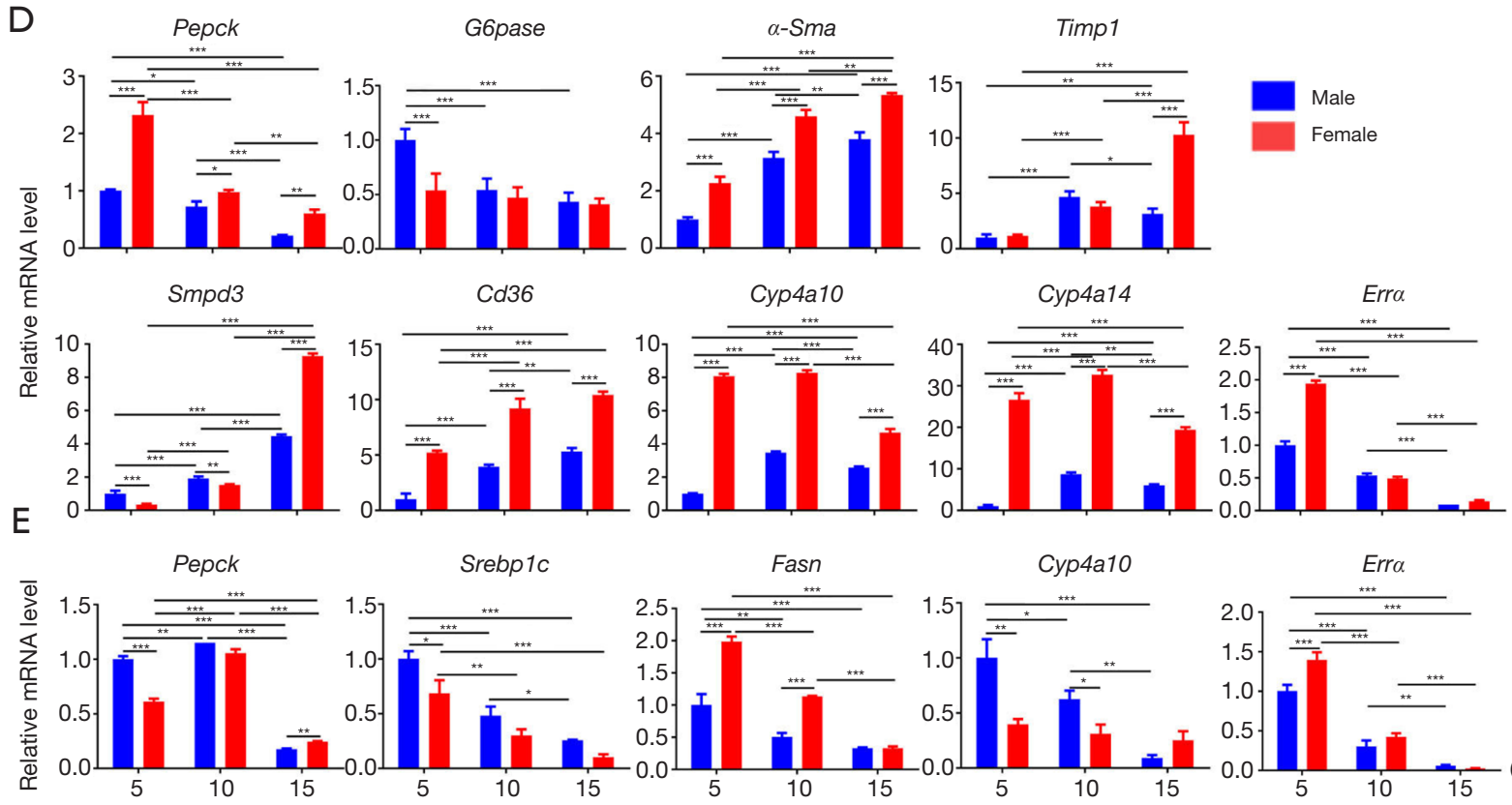

Figure 1 Aging regulates inflammatory and metabolic signaling in the livers and adipose tissue. Hepatic inflammatory gene expression (A) and protein expression (B), inflammatory gene expression in adipose tissue (C), metabolic related gene expression in liver (D) and adipose tissue (E) in 5-, 10-, and 15-month-old mice of both sexes. n=8 per group. Data are expressed as mean \pm SD. Two-way ANOVA with Tukey's correction. *, $\mathrm{P}<0.05 ;{ }^{* *}, \mathrm{P}<0.01$; ${ }^{* *}, \mathrm{P}<0.001$. Tnf $\alpha$, tumor necrosis factor alpha; Il6, interleukin 6; Il1 $\beta$, interleukin 1 beta; Saa1, serum amyloid A1; Icam1, intercellular adhesion molecule 1; Mcp1, monocyte chemoattractant protein 1; Ifn $\gamma$, interferon gamma; Mcp1, monocyte chemoattractant protein 1; Pepck, phosphoenolpyruvate carboxykinase; G6pase, glucose-6-phosphatase; $\alpha$-Sma, alpha smooth muscle actin; Timp1, tissue inhibitor of metalloproteinases 1; Smpd3, sphingomyelin phosphodiesterase 3; Srebp 1c, sterol regulatory element-binding protein 1c. 
In addition, aging elevated fibrosis related $\alpha-S m a$ (alpha smooth muscle actin) and Timp1 (tissue inhibitor of metalloproteinases 1), ceramide biosynthesis related Smpd3 (sphingomyelin phosphodiesterase 3), and fatty acid translocase $C d 36$. Female mice had higher expression levels of hepatic fatty acid omega-hydroxylase Cyp4a10 and Cyp4a14 than that of male mice. However, sex difference in the expression of Cyp4a10 and Cyp4a14 narrowed when mice were 15 months of age (Figure 1D). Moreover, in the visceral fat, aging reduced glucose and lipid metabolism regulator Srebp1c (sterol regulatory element-binding protein 1c), fatty acid synthase Fas, and Cyp4a10 suggesting dysregulated glucose and lipid metabolism (Figure 1E). Because reduced mitochondrial biogenesis is closely linked to aging, mitochondrial biogenesis-related genes were studied in the liver and adipose tissue (Figure 1D,E and Figure S1). The data showed reduced mRNA level of Sirt1 (sirtuin 1), CoxIV (cytochrome C oxidase subunit IV), Cs (citrate synthase), and Erra (estrogen related receptor alpha) in both liver and adipose tissue when mice were 15 months old (Figure S1).

\section{Aging reduces insulin sensitivity and narrows sex difference in insulin sensitivity}

Body weight gain and serum endotoxin level are important indicators for metabolism and inflammation. Our results showed that aging increased body weight and elevated serum LPS level in both sexes, with males having higher body weight and LPS level than female mice at the same age (Figure $2 A, B)$. Because aging has significant effects on glucose metabolism, fasting blood glucose level and ITT were analyzed to find out how age affects insulin sensitivity in both sexes. The data revealed 15-month-old male mice had the highest fasting blood glucose level among the studied groups (Figure 2C). Sex difference in fasting glucose was noted in 10- and 15-month-old mice. However, sex difference in insulin sensitivity was apparent especially in young mice. After insulin injection, young female mice (5 months) required glucose injection to rescue them from hypoglycemia (Figure 2D). Thus, a reduced dose was used in young mice (Figure 2E). Together, the sensitivity to insulin was much higher in females than that of males at all studied ages. Aging reduced insulin sensitivity and narrowed sex difference in insulin sensitivity (Figure 2D).

\section{Aging shifts fecal microbiota composition}

Age affected fecal microbiota profile. At phylum level, the abundance of Firmicutes was greater in males than females at 10 and 15 months of age (Figure $2 F$ ). In contrast, the abundance of Bacteroidetes was greater in females than males in 10- and 15-month-old mice as its abundance reduced when male mice aged. We and others have previously shown that increased Firmicutes to Bacteroidetes ratio is associated with obesity or consumption of the Western diet $(15,24)$. It is interesting to note that the ratio of Firmicutes to Bacteroidetes also increased when male mice aged, suggesting the similarity between aging and obesity (Figure $2 F$ ). At family level, Lachnospiraceae and Ruminococaceae, known as butyrate-generating bacteria, substantially reduced as mice age, especially in male mice. However, aging increased Erysipelotrichaceae in male mice but reduced it in females (Figure $2 G$ ).

The abundance of fecal bacterial genes was quantified to understand the global microbiota function (Figure 2H). The copy number of bile acid (BA) producing genes baif (bile acid inducible operon gene $\mathrm{J}$ ) and $b s h$ (bile salt hydrolase) was sex different especially in 5-month-old young mice. Aging substantially reduced both in females. In addition, the abundance of short-chain fatty acid (SCFA) producing genes including butyrate-generating $b c 0 A$, and propionateproducing $l c d A, m m d A$, and $p d u P$ were also sex different in young mice. However, the age-associated reduction of $l c d A$ and $p d u P$ was more apparent in males than females (Figure 2H). These data suggested that the effect of aging in altering the microbial function was sex specific.

\section{Aged fecal microbiota causes systemic inflammation and insulin resistance in a sex-dependent manner}

Because aged males were the most insulin resistant and had the highest serum LPS, AFMT was done using aged male mice as donors to establish the relationship between gut microbes and host metabolic phenotype. Aged feces collected from 20-month-old male mice were orally delivered to 3-week-old young mice of both sexes twice per week for 4 months. The results showed increased inflammatory signaling, such as TNF- $\alpha$ and IL-6, in the livers (Figure 3A,B) and/or adipose tissues (Figure 3C). However, the fold inductions were different based on the sex. Additionally, hepatic $I l 6$ and adipose Tnfa were not induced by AFMT in female mice. In addition, AFMT altered the expression of genes regulate glucose and fatty acid metabolism, as well as mitochondrial biogenesis (Figure $3 A, C$ ). Moreover, AFMT increased intestinal Fof 15 in male mice and abolished the sex difference in the 
A

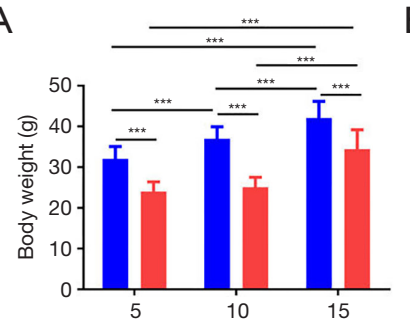

D

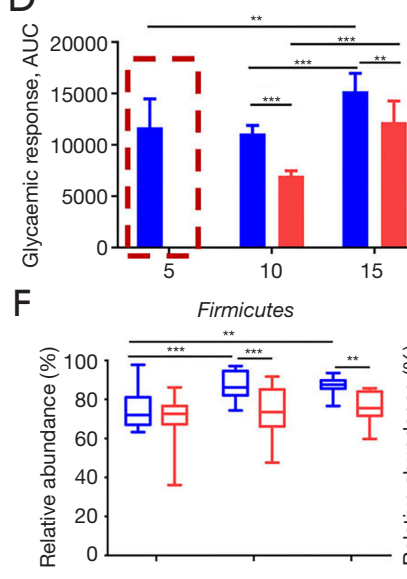

G

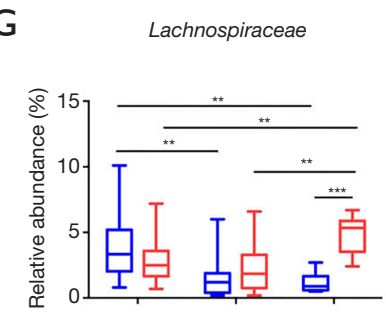

$\mathrm{H}$

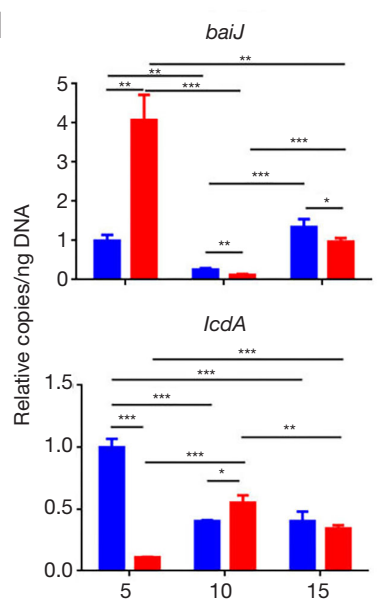

B

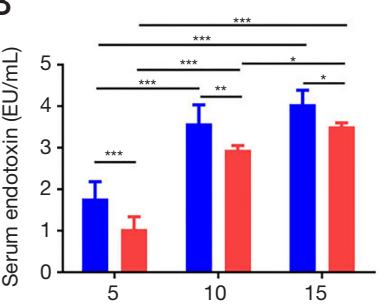

E

O
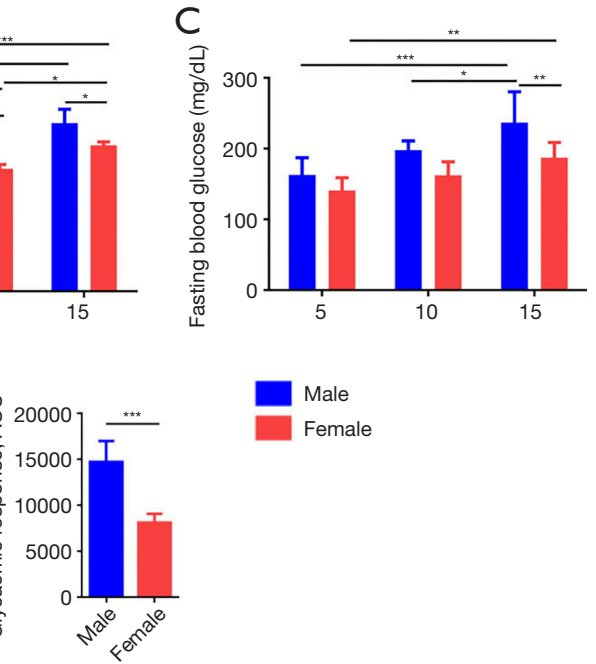

Reduce insulin dose
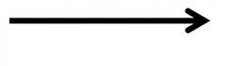

व
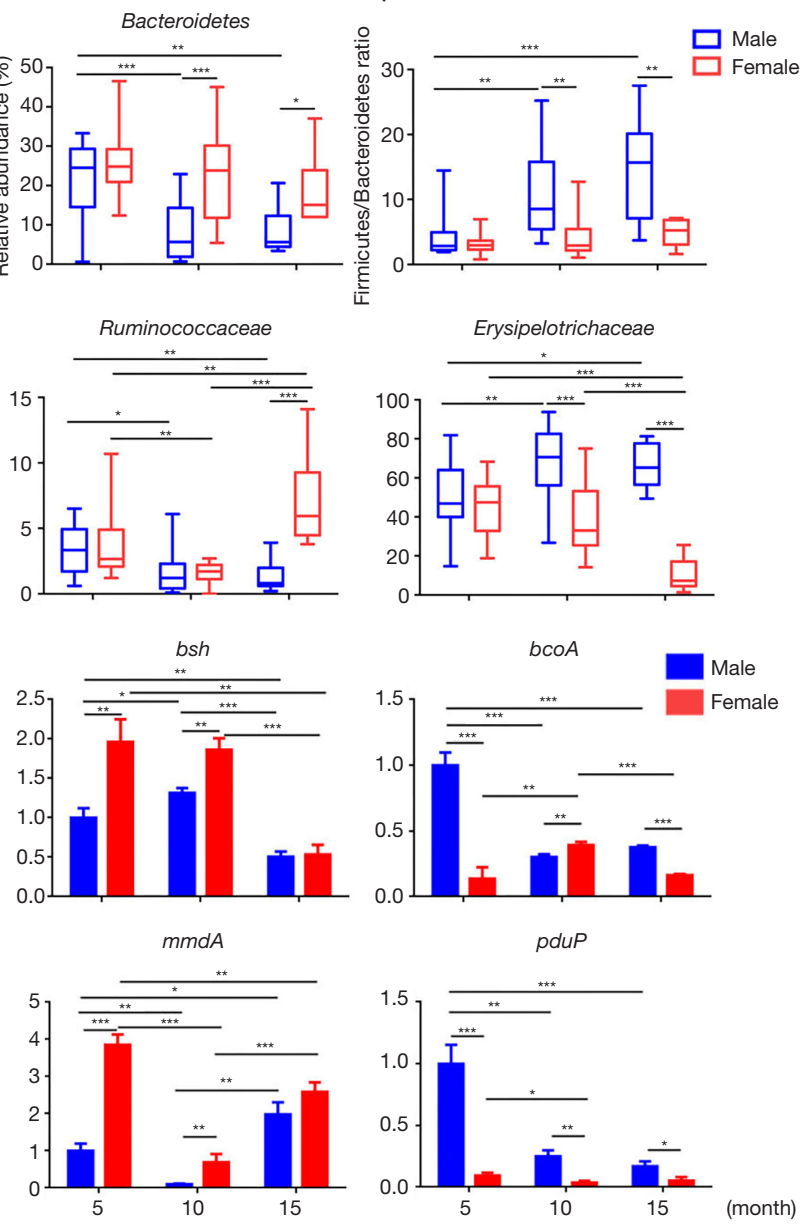

Figure 2 The changes of mouse phenotype and gut microbiota profile during aging. (A) Body weight; (B) serum endotoxin (LPS); (C) fasting blood glucose level; (D,E) area under curve (AUC) of insulin tolerance test in 5-, 10-, and 15-month-old mice of both sexes. Twoway ANOVA with Tukey's correction. (F,G) Relative abundance of fecal microbiota changes at phylum (F) and family (G) level. (H) Targeted functional quantitative PCR analysis of microbial genes. Kruskal-Wallis test. Copy number per ng DNA was calculated in each group and the relative changes to 5 -month-old male mice were shown. $\mathrm{n}=6-8$ per group. ${ }^{*}, \mathrm{P}<0.05 ;{ }^{* *}, \mathrm{P}<0.01$; ${ }^{* *}, \mathrm{P}<0.001$. baif, bile acid inducible operon gene J; bsh, bile salt hydrolase. 
A

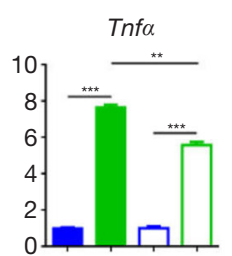

Icam1

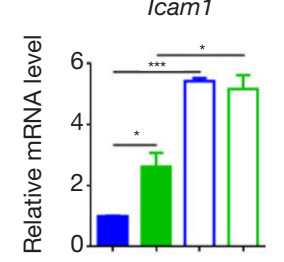

Cyp4a14

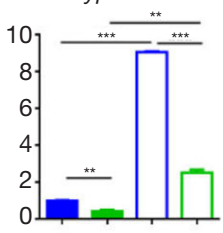

C

$\mathrm{D}$

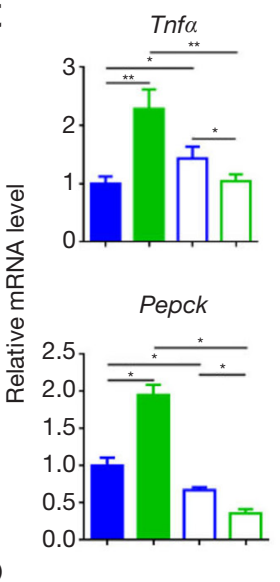

FXr

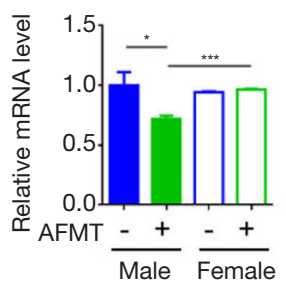

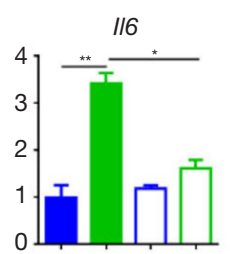

Pepck

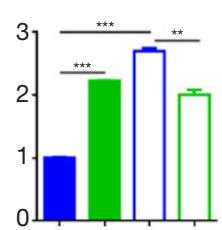

Fgf21

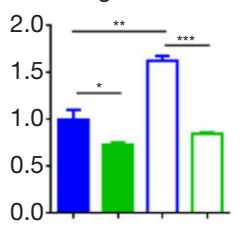

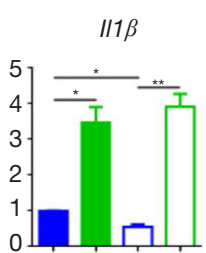

G6pase

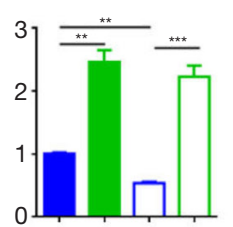

Erro

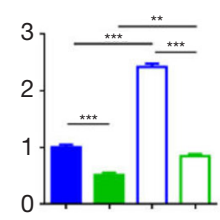

B

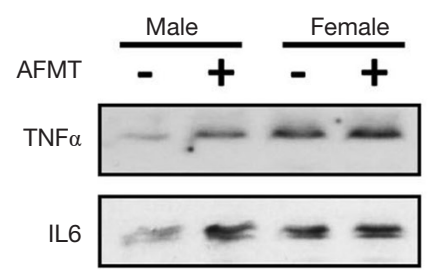

$\beta$-actin
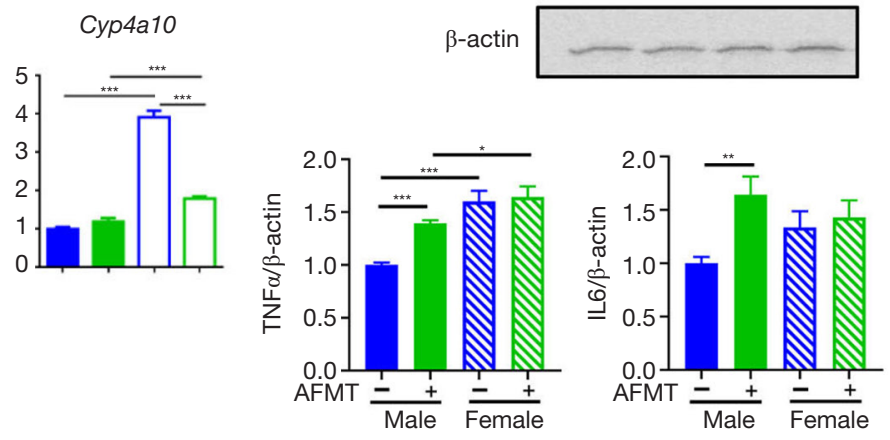
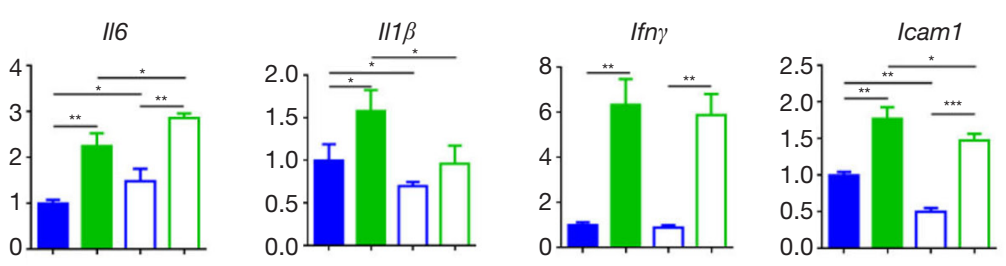

G6pase
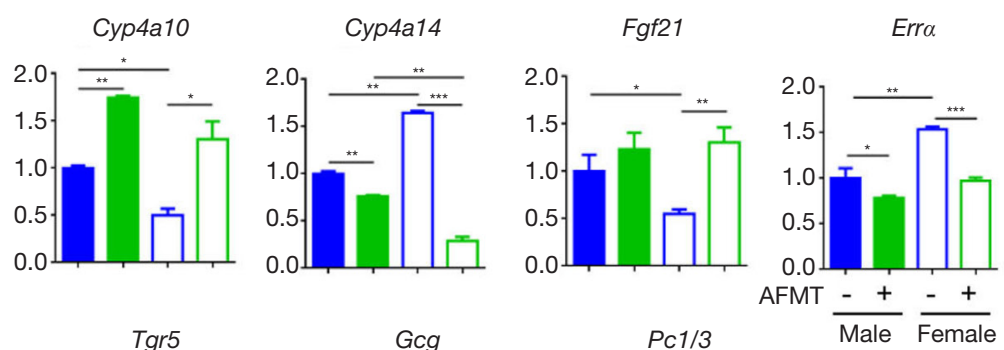

Fgf15

$\operatorname{Tgr} 5$

Gcg

$\operatorname{PC1/3}$

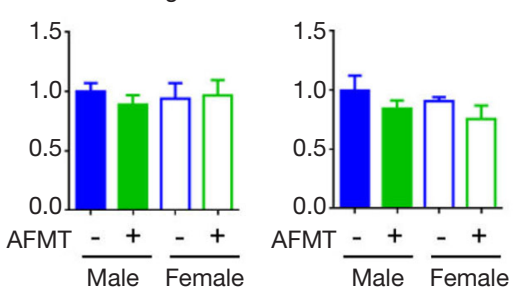

Figure 3 Transplantation of aged male fecal microbiota causes systemic inflammation and metabolic disorders. The expression of inflammation and metabolism related genes and protein in the liver (A,B), adipose tissue (C), and ileum (D) in mice of both sexes with and without aged male fecal microbiota transplantation (AFMT). $n=4$ per group. Data are expressed as mean \pm SD. One-way ANOVA with Tukey's correction. *, $\mathrm{P}<0.05$; **, $\mathrm{P}<0.01$; ${ }^{* * *}, \mathrm{P}<0.001$. Tnfa, tumor necrosis factor alpha; Il6, interleukin 6; Ill $\beta$, interleukin 1 beta; Saa1, serum amyloid A1; Icam1, intercellular adhesion molecule 1; Pepck, phosphoenolpyruvate carboxykinase; G6pase, glucose-6-phosphatase; Ifny, interferon gamma; $\operatorname{Tg} r 5$, Takeda $\mathrm{G}$ protein receptor 5. 
ileum Fgf15 mRNA level suggesting inhibiting hepatic BA synthesis as male mice age. Although the mRNA level of $\operatorname{Tg} r 5$ (Takeda $G$ protein receptor 5) was modestly reduced after AFMT, its downstream signaling including $G c g$ and $P c 1 / 3$ was not changed (Figure $3 D$ ).

AFMT had no effect on body weight and fasting blood glucose. However, serum endotoxin and ALT levels were higher in both sexes of mice that received aged male feces (Figure $4 A, B, C, D)$. Most interestingly, AFMT induced insulin resistance only in female mice, and thus abolished sex difference in insulin sensitivity (Figure 4E). Together, LPS induction in both sexes as well as inflammation signaling changes did not seem to explain the sex-difference in insulin sensitivity in response to AFMT.

AFMT shifted gut microbiota community structure in a sex-dependent manner. Alpha diversity analysis revealed AFMT only increased Chaol index in female recipients resulting in the significant sex difference in Chaol index after AFMT (Figure S2). In addition, without AFMT, male and female mice clustered separately (Figure S2C). However, after AFMT, male and female mice clustered much closer as indicated by Bray_curtis based non-metric multidimensional scaling (NMDS) analysis (Figure S2C). At family level, AFMT increased Porphyromonadaceae, Bacteroidaceae, Lachnospiraceae, Peptostreptococcaceae, and Ruminococcaceae but reduced Clostridiaceae and Bifidobacteriaceae in female recipients. It is also interesting to note that sex difference in Rikenellaceae was reversed after AFMT (Figure 4F). Moreover, AFMT reduced fecal $b s h$ in both sexes and $b c o A$ in males but had an opposite effect in changing the copy number of the baif, $l c d A$, $m m d A$, and $p d u P$ genes in two sexes (Figure $4 G$ ). Together, AFMT abolished the sex difference in the abundance of six studied bacterial genes (Figure $4 G$ ). These data suggested that AFMT abolished sex difference in insulin sensitivity accompanied by composition and functional changes of gut microbiota.

\section{Metabolic pathways regulated by aging and AFMT}

Untargeted GC-TOFMS was performed to understand the effect of aging in host metabolism. There were 212 known metabolites detected in mouse colon content. Partial least squares-discriminant analysis revealed sample clustering (Figure S3). It is apparent that two distinct clusters separated 5 - and 20-month-old male mice. Metabolic pathway analysis revealed that the most significant difference between 5 and 20-month-old groups was the metabolism of amino acids, including tyrosine, phenylalanine, tryptophan, valine, leucine, and isoleucine (Table S2). In addition, metabolism of cofactors and vitamins including ubiquinone and other terpenoid-quinone biosynthesis, pantothenate and CoA biosynthesis, and riboflavin metabolism were distinctly different between 5- and 20-month-old male mice. Chemical similarity enrichment analysis for metabolomics was also performed (20). There were 37 metabolites significantly changed because of age, and examples of those changes are shown in Figure 5A,B. The 20-month-old mice had a marked increase of sugar alcohols (xylitol, arabitol, and lyxitol), dicarboxylic acids (malic acid, fumaric acid, and adipic acid), hexose phosphates (galactose-6-phosphate and fructose-6-phosphate), and glutamates (beta-glutamic acid and $\mathrm{N}$-acetyl glutamate), but reduced aromatic amino acids (tyrosine, phenylalanine, and tryptophan). In addition, branched-chain amino acids (leucine, valine, and isoleucine), which are essential amino acids in promoting mitochondrial biogenesis and lifespan extension (25), were also reduced in aged mice.

Because altered inflammatory signaling might not able to explain why AFMT could abolish sex difference in insulin sensitivity, we analyzed metabolite changes in response to AFMT when mice were 5 months old (Figure $5 C, D$ ). Without AFMT, 12 set of metabolites differentially enriched in either sex when mice were 5 months old. For example, male mice had increased hexoses and unsaturated fatty acids. In contrast, female mice had enriched dicarboxylic acids, sugar alcohols, and cholic acids (Figure 5C). However, with AFMT, only sugar alcohol levels remained to be sex-different in age-matched 5 -month-old mice (Figure 5D). Therefore, AFMT narrowed the sex differences in metabolome as well as insulin sensitivity, and sugar alcohols including ribitol, lyxitol, and arabitol might not explain the change in insulin sensitivity. Moreover, metabolite set enrichment analysis showed that 4 out of 6 pathways, including catecholamine biosynthesis, thyroid hormone synthesis, valine, leucine and isoleucine degradation, and propionate metabolism, that shifted due to aging were also shifted by AFMT, suggesting that aged fecal microbiota had a similar effect on age-dependent metabolic changes (Figure 5E,F).

\section{Integrated relationship among phenotypes, gut microbiota, and metabolites}

The relationships between phenotypes, gut microbiota, and colon metabolites of the mice with and without 
A

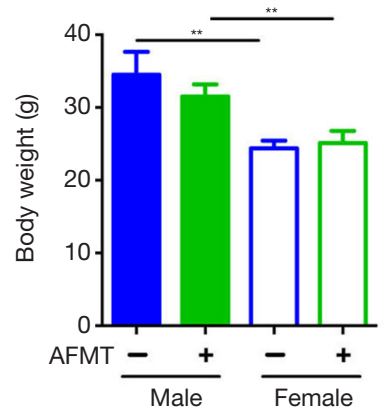

B

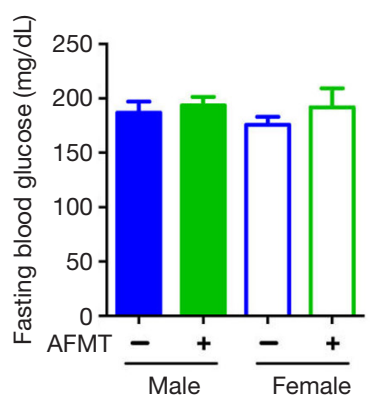

C

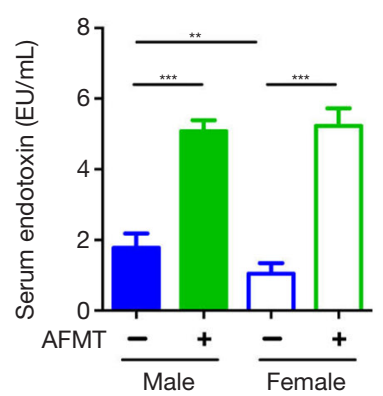

$\mathrm{D}$

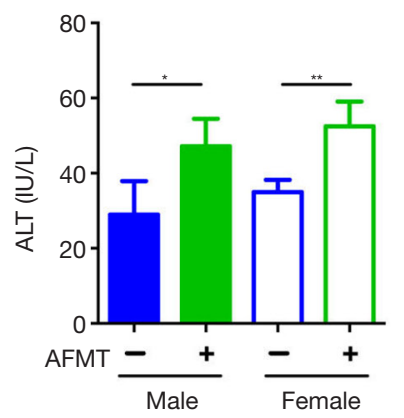

E
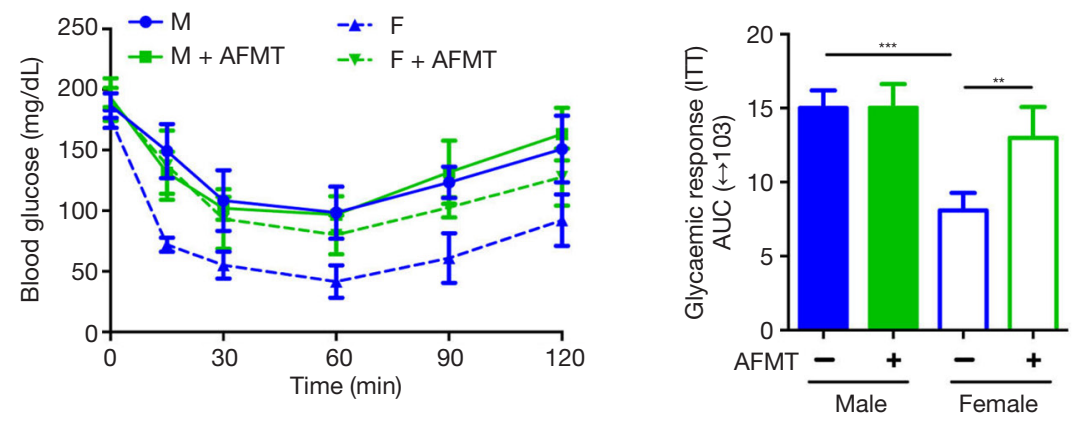

$\mathrm{F}$

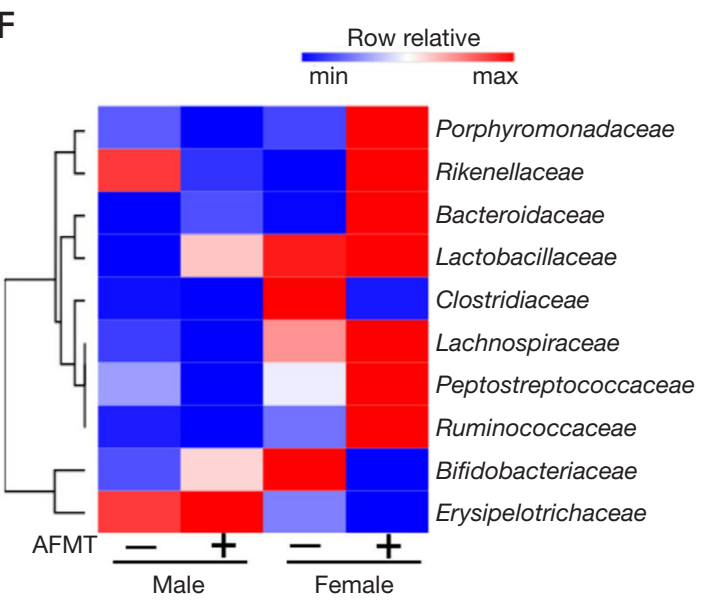

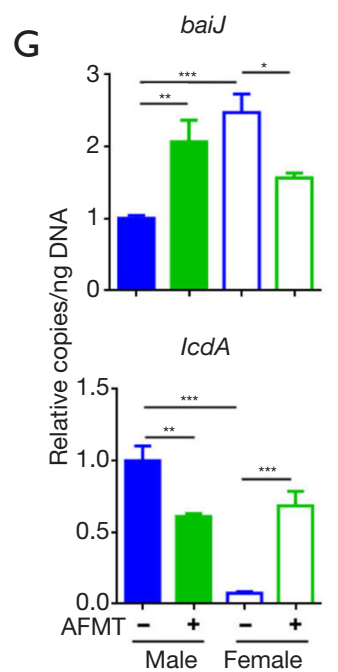
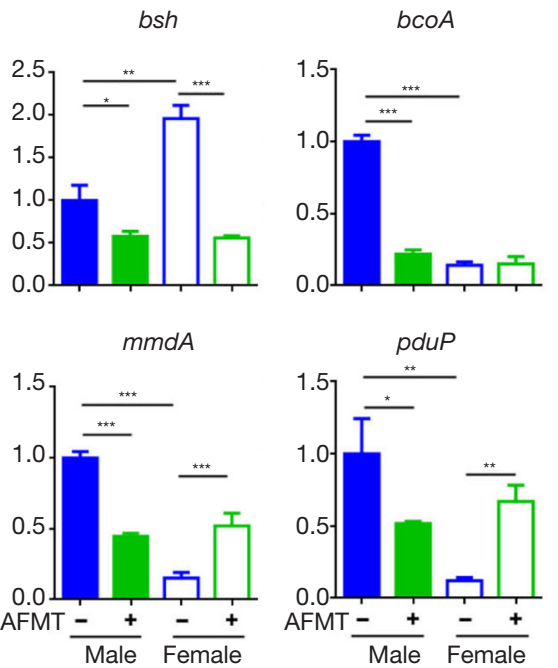

Figure 4 The changes of mouse phenotype and microbiota profile by aged male fecal microbiota transplantation (AFMT). (A) Body weight; (B) fasting blood glucose level; (C) serum endotoxin; (D) serum alanine transaminase (ALT); (E) insulin tolerance test after AFMT; (F) a heat map of fecal microbiota changes at family level; (G) targeted functional quantitative PCR analysis of microbial genes. $\mathrm{n}=4$ per group. Data are expressed as mean $\pm \mathrm{SD}$. One-way ANOVA with Tukey's correction. *, $\mathrm{P}<0.05 ;{ }^{* *}, \mathrm{P}<0.01 ;{ }^{* * *}, \mathrm{P}<0.001$. baif, bile acid inducible operon gene J; bsh, bile salt hydrolase. 

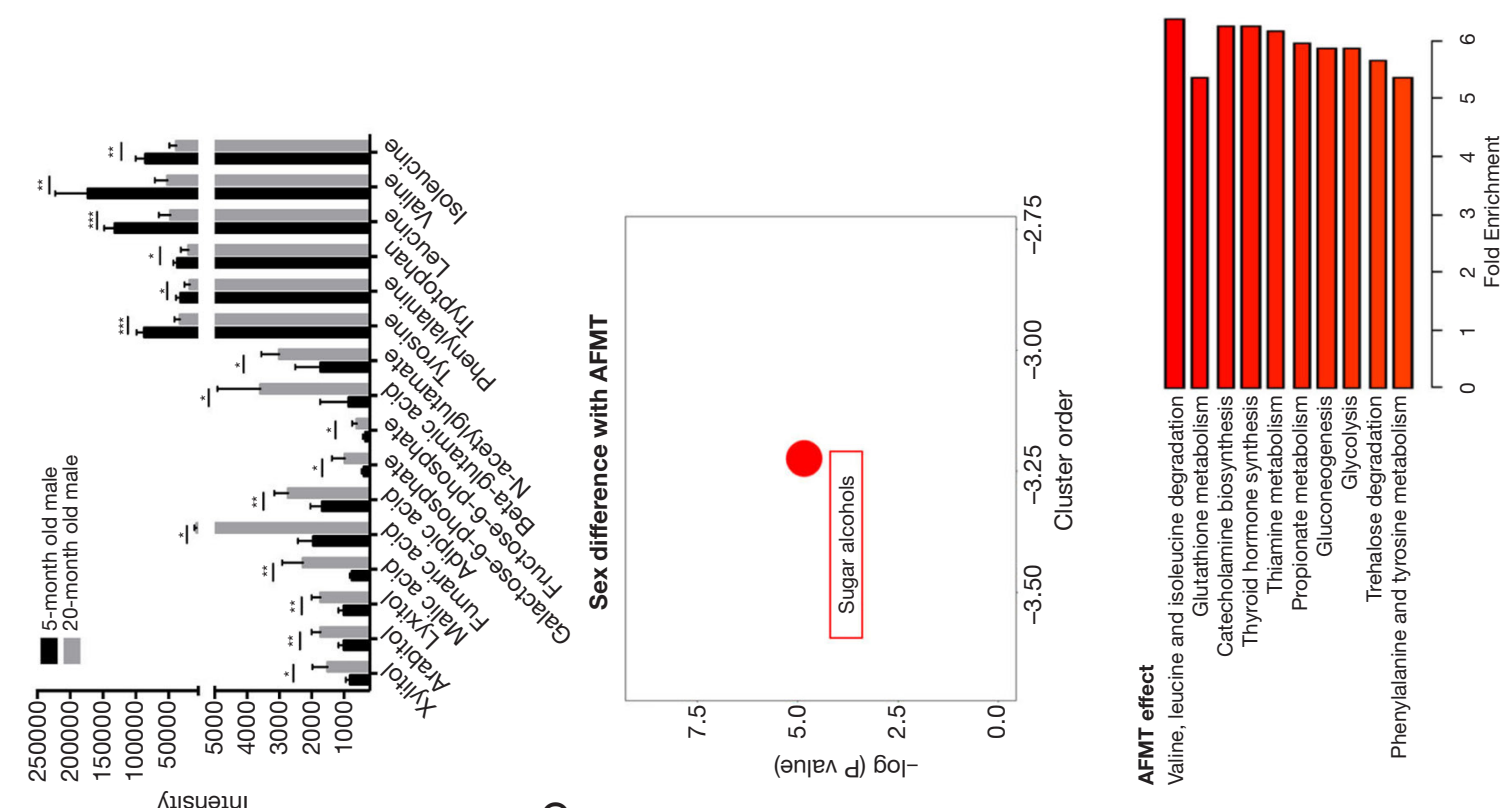

$\infty$

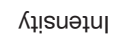

$\triangle$
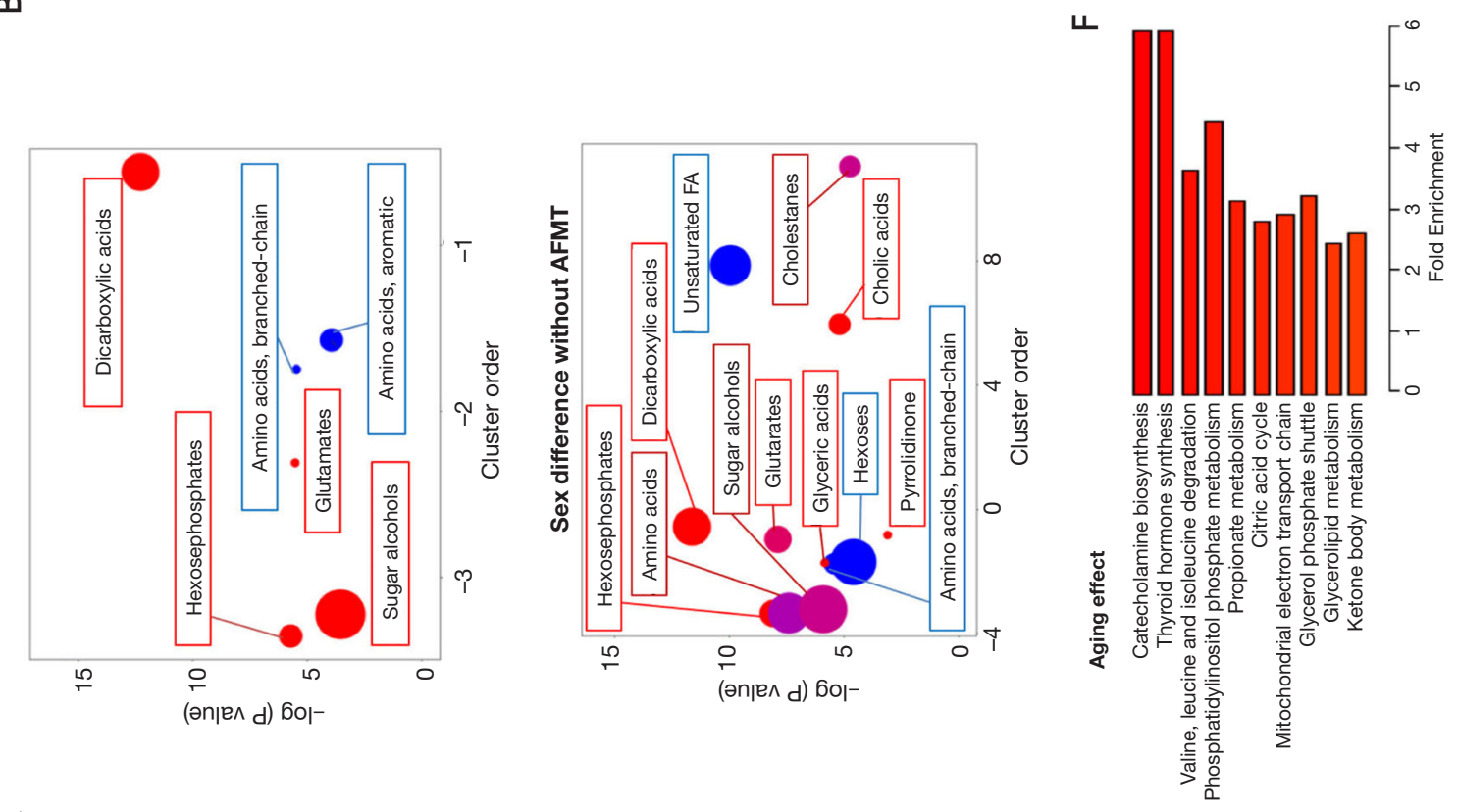

$\varangle$

U

ш 

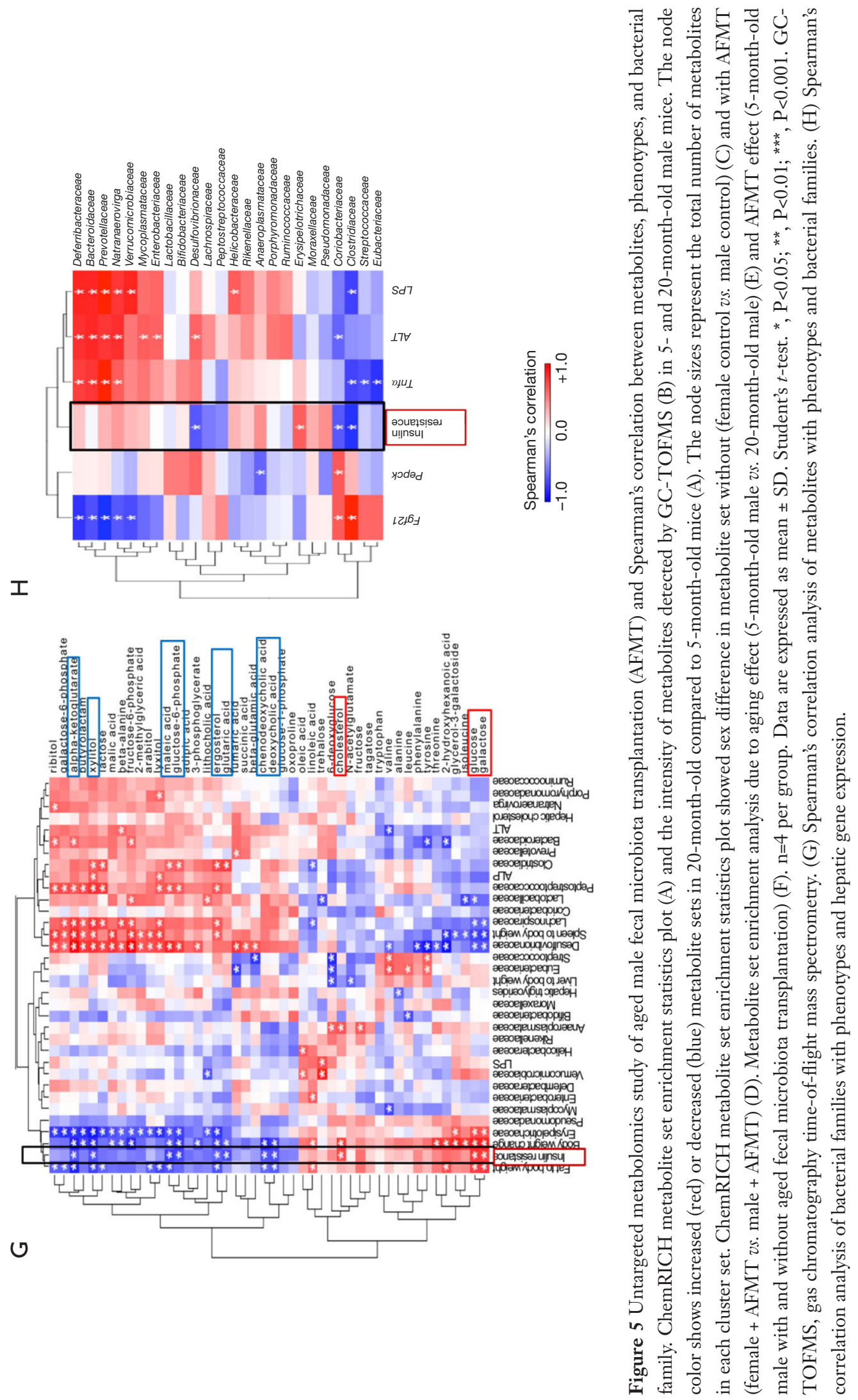
receiving AFMT were uncovered. Forty-six metabolites were significantly changed due to age, sex, or AFMT based on ChemRICH. These metabolites were used to perform Spearman's correlation analysis with 10 phenotypes and 24 bacterial families found in these mice (Figure $5 G$ ). The results showed that insulin resistance was clustered with fat-to-body weight and body weight change as well as the abundance of Erysipelotrichaceae family. In addition, insulin resistance was negatively associated with the concentration of ketone body $\alpha$-ketoglutarate, xylitol, as well as BAs including chenodeoxycholic acid (CDCA) and deoxycholic acid (DCA). Moreover, insulin resistance was positively associated with the abundance of Erysipelotrichaceae (Figure $5 H$ ). Certain bacterial families, such as Coriobacteriaceae and Clostridiaceae, were negatively associated with insulin resistance. Additionally, Coriobacteriaceae was also negatively correlated with ALT, but positively associated with hepatic Fgf21 and Pepck mRNA level. Clostridiaceae family, which was enriched in female mice but reduced after AFMT (Figure 4F), was negatively correlated with $T n f \alpha$, and LPS, while positively associated with hepatic Fgf21 mRNA level (Figure 5H).

\section{YFMT improves metabolic phenotypes in a sex-dependent manner}

Because 5-month-old female mice were the most insulinsensitive group, we studied whether fecal microbiota obtained from young females can be used to treat insulin resistance in aged mice. YFMT was performed using 2-month-old females as donors, and 10-month-old mice, which had apparent sex difference in insulin sensitivity, were used as the recipients. YFMT reduced the expression of certain inflammatory signaling genes in the livers and/ or adipose tissues (Figure 6A,B). It is interesting to note that YFMT widened the sex differences in the expression of adipose Il6, Ccl20, Il1 $\beta$, and Mcp1 genes (Figure $6 B$ ). In addition, YFMT reduced body weight and fasting blood glucose level in males, and improved insulin sensitivity in females, which further increased the sex difference in insulin sensitivity (Figure 6C,D,E).

We also studied how fecal microbiota profile changed after YFMT. YFMT didn't altered Chao1 or Shannon indices (Figure S4A,B), but enlarged the distance between male and female groups (Figure S4C). In contrast to the effect of AFMT, YFMT reduced Rikenellaceae and Peptostreptococcaceae, indicating the importance of these families on regulating insulin sensitivity in females (Figure 6F). Aging as well as AFMT reduced bsh (Figure $2 H, 4 G$ ). In contrast to AFMT, YFMT increased fecal $b s h$ in both sexes (Figure $6 G$ ). Moreover, YFMT elevated baif and SCFA-producing genes in male and/or female mice, suggesting altered gut microbiota function (Figure $6 G$ ).

Based on metabolome, only one set of metabolites, i.e., sugar alcohols had significant sex difference without YFMT when mice were 11.5 months old (Figure 6H). However, with YFMT, there were four sets of metabolites, including sugar alcohols, aromatic amino acids, dicarboxylic acids, and saturated fatty acids, were differentially enriched based on sex (Figure 6I). Thus, the enlarged sex differences in colon metabolomes might contribute to increased sex difference in insulin sensitivity in response to YFMT.

\section{Discussion}

The presented data establish the relationships between fecal microbiota and age-dependent metabolic phenotypes in both sexes. We demonstrate the efficacy of FMT in altering insulin sensitivity depends on the sex of recipients. Although YFMT reduced body weight and fasting blood glucose level in males, both young and old female mice were more susceptible to FMT-induced changes of insulin sensitivity when compared with male mice. Therefore, FMT may generate different metabolic outcomes depending on the sex of the recipient.

There is increasing interest in modulating host microbiota to treat different kinds of diseases, but very few studies have taken recipient sex into consideration. Additionally, differential colonization of gut microbiota in male and female germ-free mice has been revealed (26). Thus, it is critically important to consider the sex of both donor and recipient. Our current study shows that AFMT from male donors and YFMT from female donors can alter insulin resistance in female recipients, but not in males. These results strongly suggest that host metabolic characteristics can affect susceptibility to microbial transfer in a sex-specific manner, likely contributing to male/female disparities in susceptibility to metabolic disorders.

Inflammation is a risk factor for the development of age-associated pathologies. However, by studying a few inflammatory genes and quantification of serum LPS, there is no clear indication that the studied inflammatory signaling can fully account for the sex differences in metabolic phenotype. However, the metabolome data may be more informative than inflammatory signaling to explain the sex difference in metabolic phenotypes. 
A

B

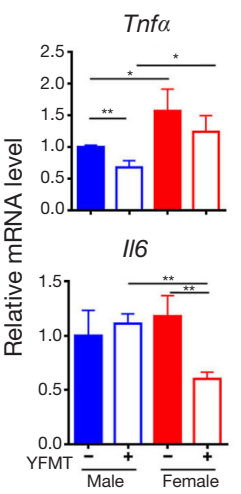

C

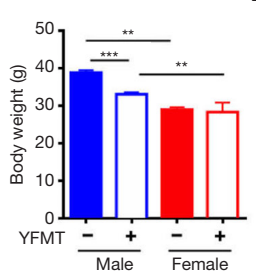

F

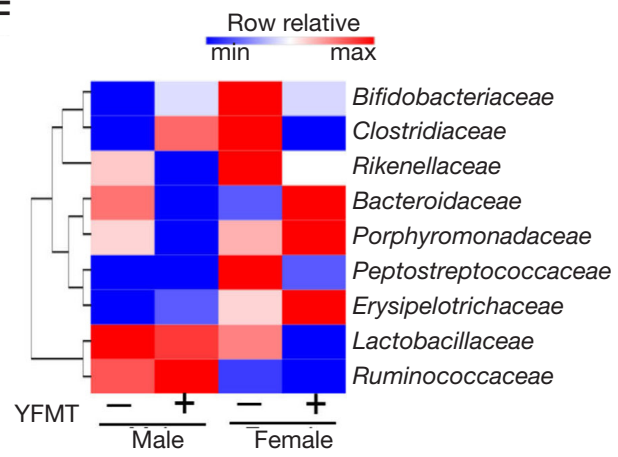

$\mathrm{H}$

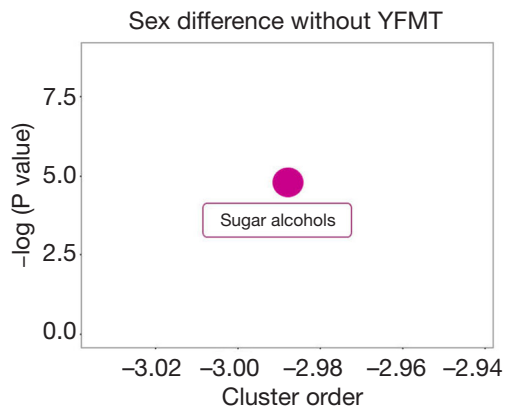

$\mathrm{Cc} / 20$

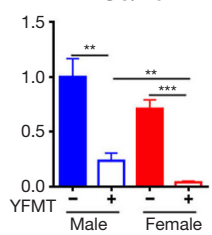

D

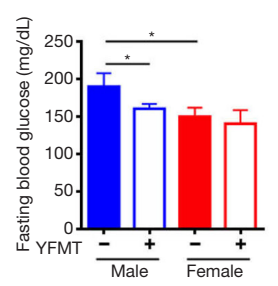

E
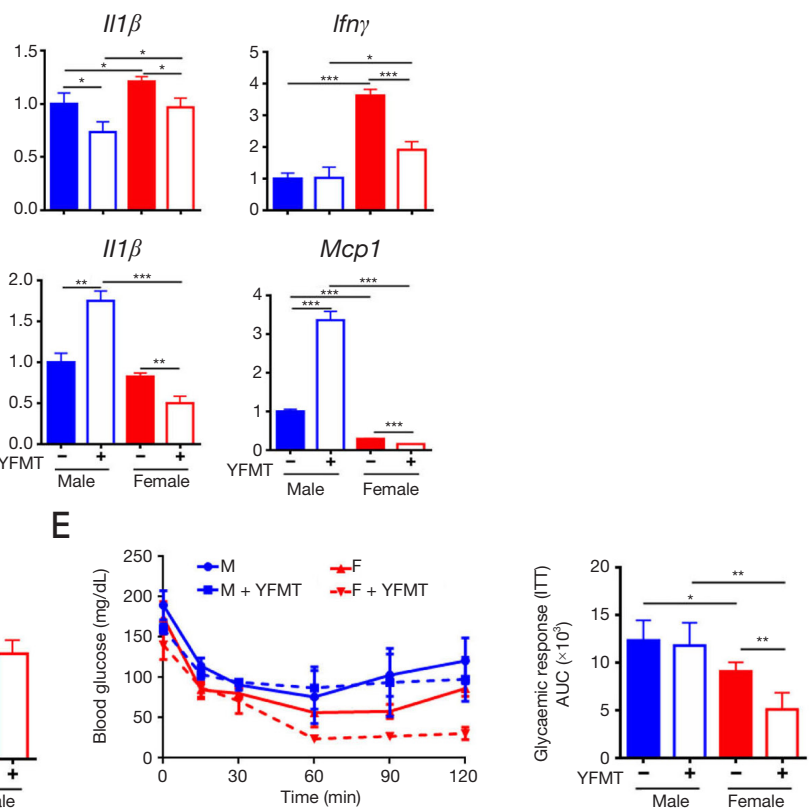

G
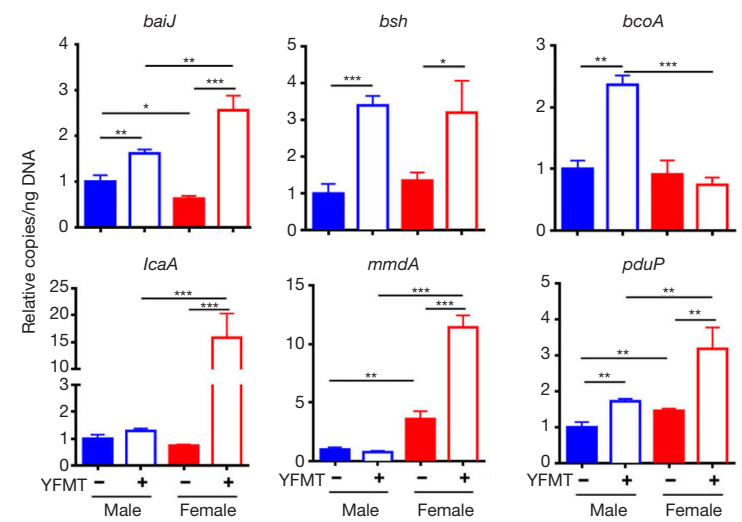

I

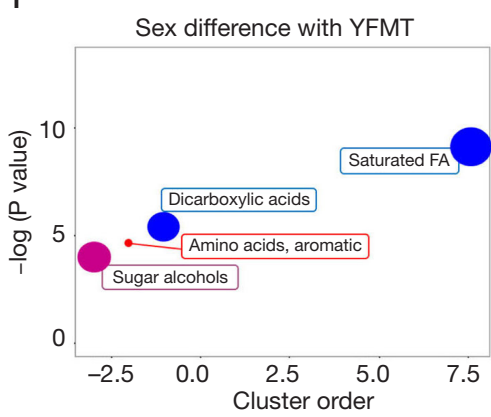

Figure 6 The changes of mouse phenotype and microbiota profile as well as untargeted metabolomics study after young female fecal microbiota transplantation (YFMT). (A) Hepatic gene expression; (B) hepatic protein level; (C) body weight; (D) fasting blood glucose level; (E) insulin tolerance test after YFMT; (F) a heat map of fecal microbiota changes at family level; (G) targeted functional quantitative PCR analysis of microbial genes. Copy number per ng DNA was calculated in each group and the relative changes to 11.5 -month-old male mice were shown. ChemRICH metabolite set enrichment statistics plot showed sex difference in metabolite set without (male control vs. female control) $(\mathrm{H})$ and with YFMT (male + YFMT $v$ s. female + YFMT) (I). $\mathrm{n}=3-4$ per group. Data are expressed as mean $\pm \mathrm{SD}$. One-way ANOVA with Tukey's correction. *, $\mathrm{P}<0.05 ;{ }^{* *}, \mathrm{P}<0.01$; ${ }^{* *}, \mathrm{P}<0.001$. Tnf $\alpha$, tumor necrosis factor alpha; Il6, interleukin 6; Il1 $\beta$, interleukin 1 beta; Ifn $\gamma$, interferon gamma; $M c p 1$, monocyte chemoattractant protein 1; baif, bile acid inducible operon gene J; bsh, bile salt hydrolase. 
Without AFMT, 5-month-old male and female mice had 12 sets of metabolites differentially found in them. For examples, males had increased colonic unsaturated fatty acids (linoleic acid) and hexoses (glucose and galactose) as well as reduced BAs (CDCA and DCA), dicarboxylic acids (maleic acid), and xylitol (Figure 5C). In addition, AFMT narrowed sex difference in those metabolomes, and the sex difference in insulin sensitivity no longer existed. It has been shown that supplementation of conjugated linoleic acid is associated with the development of insulin resistance in humans and mice $(27,28)$. In addition, high glucose is a well-known inducer for insulin resistance, and galactose is implicated in inducing age-associated insulin resistance $(29,30)$. Moreover, CDCA and DCA, which were higher in the colon content of female mice than male mice and the agonists for BA receptor FXR, can promote glucose metabolism $(31,32)$. These findings are consistent with our data that insulin resistance is positively associated with glucose and galactose, but negatively correlated with CDCA, DCA, and maleic acid (Figure 5G). After AFMT, the sex difference in metabolites narrowed and sex difference in insulin sensitivity was no longer noted. Thus, AFMT has a negative or pathological impact on young females under the experimental condition. When mice were 11.5 months old, only one set metabolite, i.e., sugar alcohols (sorbitol and glycerol) were differentially found in male and female mice (Figure 61). However, receiving YFMT, colonic dicarboxylic acids and saturated fatty acids became lower in males than females, and the sex difference in metabolic phenotypes enlarged. Consistent with previous report that amino acid metabolism decreased with age (33), we also found reduced branched-chain amino acids and aromatic amino acids in aged mice. It is interesting to note that metabolite set enrichment analysis showed that 4 out of 6 pathways were commonly influenced by aging and AFMT. Among them, it is known that branch-chain amino acids are associated with worse metabolic health including insulin resistance and type 2 diabetes mellitus (34). Catecholamine, as a monoamine neurotransmitter, has been reported to induce insulin resistance in man and animals (35). These findings suggest AFMT had a similar effect on age-dependent metabolic changes. Based on the elevated serum LPS and ALT levels, increased expression of inflammation-related genes, and dysregulated expression of glucose and lipid metabolism genes after AFMT, we speculate that reversing age-related microbiota might be a strategy to reduce age-associated metabolic disorders and age-induced systemic inflammation.

It is known that the microbiota changes drastically between infancy and adulthood, with a shift from dominance by Bifidobacterium to other genera within Firmicutes $(36,37)$ that reflects changes from primarily lactate metabolism and plant polysaccharide breakdown to carbohydrate metabolism and SCFA production. Fermentation plays a central role in the economy of the gut ecosystem. SCFAs are the major end products of fermentation. The current study uncovered that Lachnospiraceae and Ruminococaceae, which are butyrate-producing bacteria, were reduced during aging. Moreover, the copy number of bacterial $b c o A$ gene was reduced during aging and AFMT, while elevated in YFMT in a sex-dependent manner. Butyrate is the main source of nutrition for colonocytes and its deficiency impairs the gut barrier integrity. Butyrate also has anti-inflammatory effects by blocking NF- $\mathrm{BB}$ (38). The significance of butyrate in reducing gut and liver inflammation has been revealed (8). In consistency, stool butyrate level is reduced in aged populations (39). Our previous publication found that, supplementation of probiotic VSL\#3 to mice enriched the abundance of Lachnospiraceae and Ruminococcus, as well as increased the copy number of $b c o A$ gene, suggesting the role of probiotic in improving age-induced gut dysbiosis (40). Additionally, the abundance of gene that generates propionate, which reduces lipogenesis, serum cholesterol, and carcinogenesis, was reduced due to aging as well as AFMT, while increased by YFMT. Consistent with bacterial gene quantification data, the metabolite set enrichment analysis further confirmed that both aging and AFMT affected propionate metabolic pathway as revealed in Figure 5E, F.

There are several factors such as mitochondria biogenesis and BAs may account for aging- and microbiome-associated metabolic phenotype changes. The expression pattern of Erra, which responsible for mitochondria biogenesis, can be interesting. It is reduced by aging and AFMT, and the sex difference of its expression was not noted in the adipose tissue after AFMT. However, its direct role in gut microbiota-associated insulin sensitivity remains to be analyzed. BAs are no longer considered solely for lipid absorption; they are signaling molecules with diverse effects on regulating host inflammation and metabolism $(41,42)$. The abundance of BA de-conjugation gene $b s h$ was reduced during aging and by AFMT, while was increased by YFMT. In addition, both aging and FMT reduced the sex difference in $b s h$ levels. These findings were consistent with our previous report, in which reduced intestinal bsh was found in non-alcoholic steatohepatitis models in induced by diet or FXR inactivation (15). 
Bacteria function is age- and sex-dependent. Aging reduced sex difference in insulin sensitivity but enlarged the sex difference in the abundance of Erysipelotrichaceae with lowest abundance in 15 -month-old female mice. After mice were transplanted with aged male feces, the abundance of Erysipelotrichaceae was positively correlated with insulin resistance. However, young female feces improved insulin sensitivity in female mice accompanied by elevated Erysipelotrichaceae level. The family of Erysipelotrichaceae is implicated in both health benefits and disease processes. For example, Erysipelotrichaceae is linked to the reduced cholesterol metabolism and dyslipidemic phenotypes $(43,44)$. In addition, enriched Erysipelotrichaceae is observed in obese individuals and patients with colorectal cancer (45). However, Erysipelotrichaceae is involved in BA deconjugation and butyrate production $(46,47)$, and reduced Erysipelotrichaceae is also found in patients with Crohn's disease (48). Together with our findings, these results suggest that the effects of Erysipelotrichaceae might be age-, sex-, and host metabolism-dependent, and the specific species or strains under Erysipelotrichaceae with beneficial or negative effects should be uncovered by high resolution sequencing. In addition, previous report showed that, among the top 50 features that differentiate the Chinese long-living people from the younger groups, 28 operational taxonomic units belonging to Lachnospiraceae, Ruminococaceae, and Erysipelotrichaceae families (49). Consistent with these findings, our results also revealed significant alterations of these three families during aging. Based on the AFMT results, Coriobacteriaceae and Clostridiaceae were negatively correlated with insulin resistance and/or ALT and LPS. Coriobacteriaceae is reported to be linked to host energy metabolism (50). Clostridiaceae family, which was enriched in female mice but reduced after AFMT, was reduced in patients with inflammatory bowel disease (51).

The current study has a few limitations in study design. It would be interesting to compare the effect of donor sex on the efficacy of FMT. We did not do this because donors were selected based on their extreme insulin sensitivity phenotype. In addition, neither AFMT nor YFMT changed male mouse insulin sensitivity. It may be of interest to determine whether longer duration of FMT eventually affects insulin sensitivity in males.

\section{Conclusions}

Age affects gut microbiota profile, thereby altering colonic metabolites and metabolic pathways to affect metabolic phenotype. The age effect on inflammation and metabolism is transplantable, the outcomes can change based on the sex of recipient. These findings suggest that targeting ageand sex-specific microbiota community structure can be an effective approach to prevent and treat metabolic diseases.

\section{Acknowledgments}

We thank Michelle Nguyen and Leyi Wang for their contributions in editing this manuscript.

Funding: This study is supported by grants funded by the National Institutes of Health (U01CA179582, R01CA222490), the Mouse Metabolic Phenotyping Center (MMPC) Microbiome Program (30835-60) Subaward A192654, and the Natural Science Foundation of Shanghai (20ZR1453900).

\section{Footnote}

Reporting Checklist: The authors have completed the ARRIVE reporting checklist. Available at https://hbsn. amegroups.com/article/view/10.21037/hbsn-20-671/rc

Data Sharing Statement: Available at https://hbsn. amegroups.com/article/view/10.21037/hbsn-20-671/dss

Conflicts of Interest: All authors have completed the ICMJE uniform disclosure form (available at https://hbsn. amegroups.com/article/view/10.21037/hbsn-20-671/coif). Dr. YJYW serves as an unpaid editorial board member of Hepatobiliary Surgery and Nutrition. The other authors have no conflicts of interest to declare.

Ethical Statement: The authors are accountable for all aspects of the work in ensuring that questions related to the accuracy or integrity of any part of the work are appropriately investigated and resolved. Experiments were performed under protocols (NO. 18274) granted by the Institutional Animal Care and Use Committee of the University of California, Davis, in compliance with the National Institutes of Health Guidelines for the Care and Use of Laboratory Animals.

Open Access Statement: This is an Open Access article distributed in accordance with the Creative Commons Attribution-NonCommercial-NoDerivs 4.0 International License (CC BY-NC-ND 4.0), which permits the non- 
commercial replication and distribution of the article with the strict proviso that no changes or edits are made and the original work is properly cited (including links to both the formal publication through the relevant DOI and the license). See: https://creativecommons.org/licenses/by-nc-nd/4.0/.

\section{References}

1. Boulange CL, Neves AL, Chilloux J, et al. Impact of the gut microbiota on inflammation, obesity, and metabolic disease. Genome Med 2016;8:42.

2. Wang L, Wan YY. The role of gut microbiota in liver disease development and treatment. Liver Res 2019;3:3-18.

3. Rea IM, Gibson DS, McGilligan V, et al. Age and AgeRelated Diseases: Role of Inflammation Triggers and Cytokines. Front Immunol 2018;9:586.

4. Belikov AV. Age-related diseases as vicious cycles. Ageing Res Rev 2019;49:11-26.

5. Vaiserman AM, Koliada AK, Marotta F. Gut microbiota: A player in aging and a target for anti-aging intervention. Ageing Res Rev 2017;35:36-45.

6. Thevaranjan N, Puchta A, Schulz C, et al. Age-Associated Microbial Dysbiosis Promotes Intestinal Permeability, Systemic Inflammation, and Macrophage Dysfunction. Cell Host Microbe 2017;21:455-466.e4. Erratum in: Cell Host Microbe. 2018 Apr 11;23(4):570. doi: 10.1016/ j.chom.2018.03.006.

7. Gentile CL, Weir TL. The gut microbiota at the intersection of diet and human health. Science 2018;362:776-80.

8. Sheng L, Jena PK, Hu Y, et al. Hepatic inflammation caused by dysregulated bile acid synthesis is reversible by butyrate supplementation. J Pathol 2017;243:431-41.

9. Jena PK, Sheng L, Nagar N, et al. Synbiotics Bifidobacterium infantis and milk oligosaccharides are effective in reversing cancer-prone nonalcoholic steatohepatitis using western diet-fed FXR knockout mouse models. J Nutr Biochem 2018;57:246-54.

10. Barcena C, Valdes-Mas R, Mayoral P, et al. Healthspan and lifespan extension by fecal microbiota transplantation into progeroid mice. Nat Med 2019;25:1234-42.

11. de Groot P, Scheithauer T, Bakker GJ, et al. Donor metabolic characteristics drive effects of faecal microbiota transplantation on recipient insulin sensitivity, energy expenditure and intestinal transit time. Gut 2020;69:502-12.

12. Vrieze A, Van Nood E, Holleman F, et al. Transfer of intestinal microbiota from lean donors increases insulin sensitivity in individuals with metabolic syndrome. Gastroenterology 2012;143:913-6.e7. Erratum in: Gastroenterology. 2013 Jan;144(1):250.

13. Kootte RS, Levin E, Salojarvi J, et al. Improvement of Insulin Sensitivity after Lean Donor Feces in Metabolic Syndrome Is Driven by Baseline Intestinal Microbiota Composition. Cell Metab 2017;26:611-619.e6.

14. Geer EB, Shen W. Gender differences in insulin resistance, body composition, and energy balance. Gend Med 2009;6 Suppl 1:60-75.

15. Sheng L, Jena PK, Liu HX, et al. Gender Differences in Bile Acids and Microbiota in Relationship with Gender Dissimilarity in Steatosis Induced by Diet and FXR Inactivation. Sci Rep 2017;7:1748.

16. Fransen F, van Beek AA, Borghuis T, et al. The Impact of Gut Microbiota on Gender-Specific Differences in Immunity. Front Immunol 2017;8:754.

17. Vemuri R, Sylvia KE, Klein SL, et al. The microgenderome revealed: sex differences in bidirectional interactions between the microbiota, hormones, immunity and disease susceptibility. Semin Immunopathol 2019;41:265-75.

18. Ryan MJ, Schloter M, Berg G, et al. Development of Microbiome Biobanks - Challenges and Opportunities. Trends Microbiol 2021;29:89-92.

19. Fiehn O, Wohlgemuth G, Scholz M, et al. Quality control for plant metabolomics: reporting MSI-compliant studies. Plant J 2008;53:691-704.

20. Barupal DK, Fiehn O. Chemical Similarity Enrichment Analysis (ChemRICH) as alternative to biochemical pathway mapping for metabolomic datasets. Sci Rep 2017;7:14567.

21. Frese SA, Parker K, Calvert CC, et al. Diet shapes the gut microbiome of pigs during nursing and weaning. Microbiome 2015;3:28.

22. Sheng L, Jena PK, Liu HX, et al. Obesity treatment by epigallocatechin-3-gallate-regulated bile acid signaling and its enriched Akkermansia muciniphila. FASEB J 2018;32:6371-84.

23. Jena PK, Sheng L, Liu HX, et al. Western Diet-Induced Dysbiosis in Farnesoid X Receptor Knockout Mice Causes Persistent Hepatic Inflammation after Antibiotic Treatment. Am J Pathol 2017;187:1800-13. 
24. Ley RE, Turnbaugh PJ, Klein S, et al. Microbial ecology: human gut microbes associated with obesity. Nature 2006;444:1022-3.

25. Valerio A, D'Antona G, Nisoli E. Branched-chain amino acids, mitochondrial biogenesis, and healthspan: an evolutionary perspective. Aging (Albany NY) 2011;3:464-78.

26. Wang JJ, Wang J, Pang XY, et al. Sex differences in colonization of gut microbiota from a man with shortterm vegetarian and inulin-supplemented diet in germ-free mice. Sci Rep 2016;6:36137.

27. Riserus U, Vessby B, Arnlov J, et al. Effects of cis9,trans-11 conjugated linoleic acid supplementation on insulin sensitivity, lipid peroxidation, and proinflammatory markers in obese men. Am J Clin Nutr 2004;80:279-83.

28. Tsuboyama-Kasaoka N, Takahashi M, Tanemura K, et al. Conjugated linoleic acid supplementation reduces adipose tissue by apoptosis and develops lipodystrophy in mice. Diabetes 2000;49:1534-42.

29. Nelson BA, Robinson KA, Buse MG. High glucose and glucosamine induce insulin resistance via different mechanisms in 3T3-L1 adipocytes. Diabetes 2000;49:981-91.

30. Kenawy S, Hegazy R, Hassan A, et al. Involvement of insulin resistance in D-galactose-induced age-related dementia in rats: Protective role of metformin and saxagliptin. PLoS One 2017;12:e0183565.

31. Chiang JYL, Ferrell JM. Bile Acids as Metabolic Regulators and Nutrient Sensors. Annu Rev Nutr 2019;39:175-200.

32. Ma J, Hong $\mathrm{Y}$, Zheng $\mathrm{N}$, et al. Gut microbiota remodeling reverses aging-associated inflammation and dysregulation of systemic bile acid homeostasis in mice sex-specifically. Gut Microbes 2020;11:1450-74.

33. Usui Y, Kimura Y, Satoh T, et al. Effects of long-term intake of a yogurt fermented with Lactobacillus delbrueckii subsp. bulgaricus 2038 and Streptococcus thermophilus 1131 on mice. Int Immunol 2018;30:319-31.

34. Lynch CJ, Adams SH. Branched-chain amino acids in metabolic signalling and insulin resistance. Nat Rev Endocrinol 2014;10:723-36.

35. Kirsch DM, Baumgarten M, Deufel T, et al. Catecholamine-induced insulin resistance of glucose transport in isolated rat adipocytes. Biochem J 1983;216:737-45.

36. Yatsunenko T, Rey FE, Manary MJ, et al. Human gut microbiome viewed across age and geography. Nature 2012;486:222-7.

37. Mariat D, Firmesse O, Levenez F, et al. The Firmicutes/ Bacteroidetes ratio of the human microbiota changes with age. BMC Microbiol 2009;9:123.

38. Hamer HM, Jonkers D, Venema K, et al. Review article: the role of butyrate on colonic function. Aliment Pharmacol Ther 2008;27:104-19.

39. Woodmansey EJ, McMurdo ME, Macfarlane GT, et al. Comparison of compositions and metabolic activities of fecal microbiotas in young adults and in antibiotic-treated and non-antibiotic-treated elderly subjects. Appl Environ Microbiol 2004;70:6113-22.

40. Jena PK, Sheng L, Li Y, et al. Probiotics VSL\#3 are effective in reversing non-alcoholic steatohepatitis in a mouse model. Hepatobiliary Surg Nutr 2020;9:170-82.

41. Li T, Chiang JY. Bile acid signaling in metabolic disease and drug therapy. Pharmacol Rev 2014;66:948-83.

42. Liu HX, Keane R, Sheng L, et al. Implications of microbiota and bile acid in liver injury and regeneration. J Hepatol 2015;63:1502-10.

43. Spencer MD, Hamp TJ, Reid RW, et al. Association between composition of the human gastrointestinal microbiome and development of fatty liver with choline deficiency. Gastroenterology 2011;140:976-86.

44. Martinez I, Wallace G, Zhang C, et al. Diet-induced metabolic improvements in a hamster model of hypercholesterolemia are strongly linked to alterations of the gut microbiota. Appl Environ Microbiol 2009;75:4175-84.

45. Kaakoush NO. Insights into the Role of Erysipelotrichaceae in the Human Host. Front Cell Infect Microbiol 2015;5:84.

46. Vital M, Howe AC, Tiedje JM. Revealing the bacterial butyrate synthesis pathways by analyzing (meta)genomic data. mBio 2014;5:e0889.

47. Labbe A, Ganopolsky JG, Martoni CJ, et al. Bacterial bile metabolising gene abundance in Crohn's, ulcerative colitis and type 2 diabetes metagenomes. PLoS One 2014;9:e115175.

48. Gevers D, Kugathasan S, Denson LA, et al. The treatment-naive microbiome in new-onset Crohn's disease. Cell Host Microbe 2014;15:382-92.

49. Kong F, Hua Y, Zeng B, et al. Gut microbiota signatures of longevity. Curr Biol 2016;26:R832-33.

50. Claus SP, Ellero SL, Berger B, et al. Colonization- 
induced host-gut microbial metabolic interaction. mBio 2011;2:e00271-10.

51. Imhann F, Vich Vila A, Bonder MJ, et al. Interplay of host genetics and gut microbiota underlying the onset and clinical presentation of inflammatory bowel disease. Gut 2018;67:108-19.

Cite this article as: Sheng L, Jena PK, Hu Y, Wan YJY. Agespecific microbiota in altering host inflammatory and metabolic signaling as well as metabolome based on the sex. HepatoBiliary Surg Nutr 2021;10(1):31-48. doi: 10.21037/hbsn-20-671 
Table S1 List of primers used in this study

\begin{tabular}{|c|c|c|}
\hline Gene & $5^{\prime}-3^{\prime}$ sequence & Reference \\
\hline \multirow[t]{2}{*}{ Tnfa } & F: TCGAGTGACAAGCCTGTAG & \\
\hline & R: GTTGGTTGTCTTTGAGATCC & \\
\hline \multirow[t]{2}{*}{116} & F: GTTGCCTTCTTGGGACTGATG & \\
\hline & R: GGGAGTGGTATCCTCTGTGAAGTCT & \\
\hline \multirow[t]{2}{*}{$\| 1 \beta$} & F: AAGATGAAGGGCTGCTTCCA & \\
\hline & R: GTGCTGCTGCGAGATTTGAA & \\
\hline \multirow[t]{2}{*}{ Saa1 } & F: GACAAATACTGAGCGTCCTC & \\
\hline & R: ATTACССТСТССТССТCAAG & \\
\hline \multirow[t]{2}{*}{ Icam1 } & F: CAGTCCGCTGTGCTITGAGA & \\
\hline & R: CGGAAACGAATACACGGTGAT & \\
\hline \multirow[t]{2}{*}{ Mcp1 } & F: GCAGTTAACGCCCCACTCA & \\
\hline & R: CCCAGCCTACTCATTGGGATCA & \\
\hline \multirow[t]{2}{*}{ Ifn $\gamma$} & F: AGCTCTTCCTCATGGCTGTT & \\
\hline & R: TCCTTTTGCCAGTTCCTCCA & \\
\hline \multirow[t]{2}{*}{ Pepck } & F: TGTCGGAAGAAGACTTTGAGAAA & \\
\hline & R: TGCTGAATGGGATGACATACATG & \\
\hline \multirow[t]{2}{*}{ G6pase } & F: GGAGTCTTGTCAGGCATTGCT & \\
\hline & R: GCGCGAAACCAAACAAGAAG & \\
\hline \multirow[t]{2}{*}{$\alpha-S m a$} & F: ACTGGTATTGTGCTGGACTC & \\
\hline & R: CATGAGGTAGTCGGTGAGAT & \\
\hline \multirow[t]{2}{*}{ Timp1 } & F: CCTGGTCATAAGGGCTAAATTCA & \\
\hline & R: TTAGTCATCTTGATCTTATAACGCTGC & \\
\hline \multirow[t]{2}{*}{ Smpd3 } & F: TCATGGACGTGGCCTATCACTGTT & \\
\hline & R: TGCAGGCGATGTACCCAACAATTC & \\
\hline \multirow[t]{2}{*}{ Cd36 } & F: AGGTCTATCTACGCTGTGTTCGGA & \\
\hline & R: CAATGGTTGTCTGGATTCTGGAGGG & \\
\hline \multirow[t]{2}{*}{ Сур4a10 } & F: TCCAGGTTTGCACCAGACTCT & \\
\hline & R: TCCTGGCTCCTCCTGAGAAG & \\
\hline \multirow[t]{2}{*}{ Cyp4a14 } & F: CAAGACCCTCCAGCATTTCC & \\
\hline & R: GAGCTCCTTGTCCTTCAGATGGT & \\
\hline \multirow[t]{2}{*}{ Erra } & F: TCAAGGAGGGTGTGCGTCTG & \\
\hline & R: CTTGGCCCAGCTGATGGTGA & \\
\hline \multirow[t]{2}{*}{ Srebp1c } & F: TGTGATCTACTTCTTGTGGCCCGT & \\
\hline & R: AGGCTGCTCAGGTCATGTTGGAAA & \\
\hline Fasn & F: GGTGTGGTGGGTTGGTGAATTGT & \\
\hline & R: TGCTTTAGCACCTGCTGTAGCTCT & \\
\hline Fgf21 & F: CTGGGGGTCTACCAAGCATA & \\
\hline & R: CACCCAGGATTTGAATGACC & \\
\hline Fxr & F: TGCTCACAGCGATCGTCATC & \\
\hline & R: TCTCAGCGTGGTGATGGTTG & \\
\hline Fgf15 & F: TGAAGACGATTGCCATCAAG & \\
\hline & R: GAGTAGCGAATCAGCCCGTA & \\
\hline $\operatorname{Tgr} 5$ & F: CTGCCCAAAGGTGTCTACGA & \\
\hline & R: GCATTGGCTACTGGTGTGGT & \\
\hline Gcg & F: TGAGATGAGCACCATTCTGGA & \\
\hline & R: TCCGCAGAGATGTTGTGAAGA & \\
\hline$P c 1 / 3$ & F: TCAACCAGAGCACATGAAGC & \\
\hline & R: ATTGCTGCTGCTGGAGTTTT & \\
\hline Functional & & \\
\hline baiJ & F: TCAGGACGTGGAGGCGATCCA & (52) \\
\hline & R: TACRTGATACTGGTAGCTCCA & \\
\hline bsh & F: ATGGGCGGACTAGGATTACC & (53) \\
\hline & R: TGCCACTCTCTGTCTGCATC & \\
\hline$b c o A$ & F: GCIGAICATTTCACITGGAAYWSITGGCAYATG & (54) \\
\hline & R: CCTGCCTTTGCAATRTCIACRAANGC & \\
\hline pduP & F: GTGGATGARACIGGIATGGGNAAYGTNGG & (55) \\
\hline & R: CAATAGCCYTCICCICCRAAICCIADNGC & \\
\hline$m m d A$ & F: AATGACTCGGGIGGIGCIMGNATHCARGA & (55) \\
\hline & R: GATTGTTACYTTIGGIACNGTNGCYTC & \\
\hline$I c d A$ & F: CTGGTGTGCTGGWSIGCIWSIGTIGCNCC & (55) \\
\hline & R: CAGATAGGTCCAIAYIGCDATNCCYTCCCA & \\
\hline
\end{tabular}




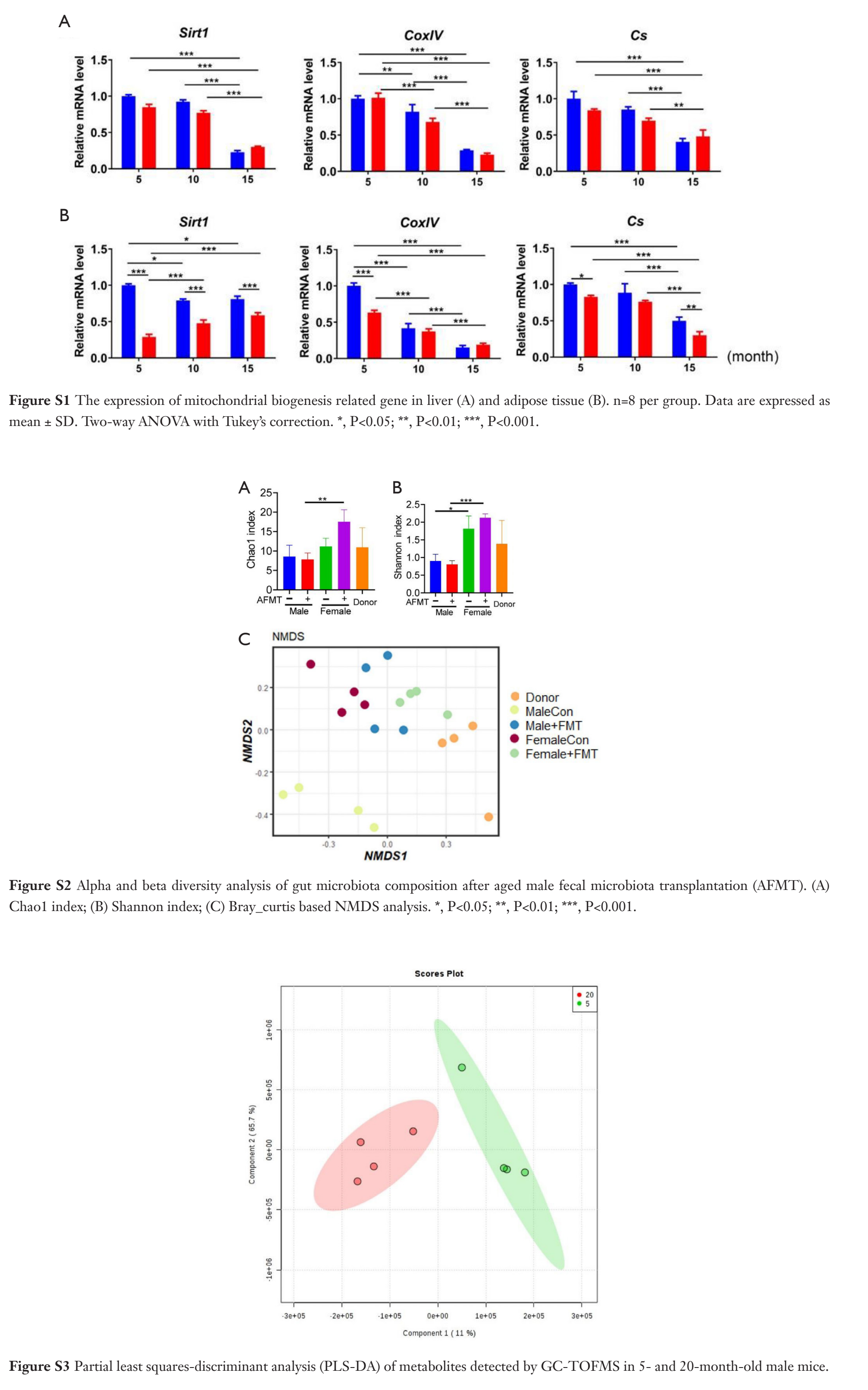


Table S2 Metabolic pathway and function analysis between 5- and 20-month-old male mice

\begin{tabular}{|c|c|c|c|c|}
\hline Pathway name & $\begin{array}{l}\text { Hits/total } \\
\text { compounds }\end{array}$ & $\mathrm{P}$ & FDR & Function \\
\hline $\begin{array}{l}\text { Ubiquinone and other terpenoid- } \\
\text { quinone biosynthesis }\end{array}$ & $1 / 3$ & 0.000243 & 0.004595 & Metabolism of cofactors and vitamins \\
\hline Tyrosine metabolism & $3 / 44$ & 0.000286 & 0.004595 & Amino acid metabolism \\
\hline $\begin{array}{l}\text { Phenylalanine, tyrosine and tryptophan } \\
\text { biosynthesis }\end{array}$ & $2 / 4$ & 0.000312 & 0.004595 & Amino acid metabolism \\
\hline Phenylalanine metabolism & $4 / 11$ & 0.000312 & 0.004595 & Amino acid metabolism \\
\hline $\begin{array}{l}\text { Valine, leucine and isoleucine } \\
\text { biosynthesis }\end{array}$ & $4 / 11$ & 0.001602 & 0.015756 & Amino acid metabolism \\
\hline $\begin{array}{l}\text { Valine, leucine and isoleucine } \\
\text { degradation }\end{array}$ & $4 / 38$ & 0.001602 & 0.015756 & Amino acid metabolism \\
\hline Pantothenate and CoA biosynthesis & $4 / 15$ & 0.003982 & 0.033561 & Metabolism of cofactors and vitamins \\
\hline Citrate cycle (TCA cycle) & $5 / 20$ & 0.036678 & 0.2705 & Carbohydrate metabolism \\
\hline Tryptophan metabolism & $2 / 40$ & 0.042778 & 0.28043 & Amino acid metabolism \\
\hline Riboflavin metabolism & $1 / 11$ & 0.049989 & 0.29494 & Metabolism of cofactors and vitamins \\
\hline
\end{tabular}

Top 10 pathways are listed.

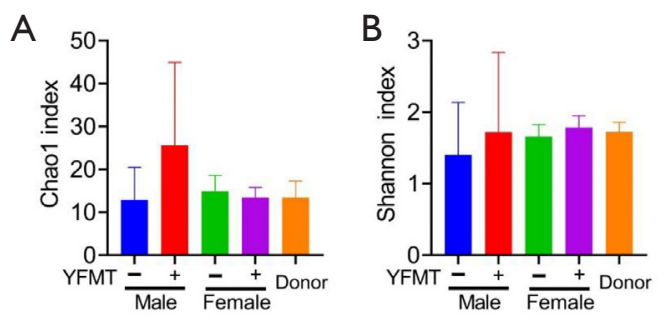

C

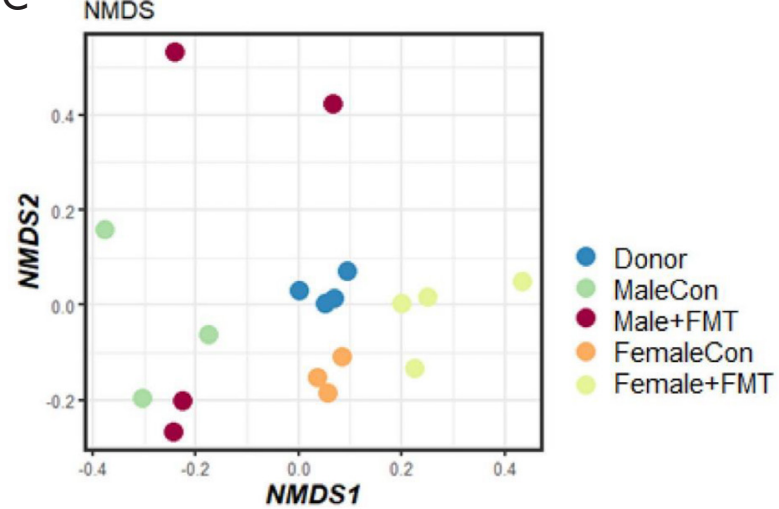

Figure S4 Alpha and beta diversity analysis of gut microbiota composition after young female fecal microbiota transplantation (YFMT). (A) Chao1 index; (B) Shannon index; (C) Bray_curtis based NMDS analysis.

\section{References}

52. Yoshimoto S, Loo TM, Atarashi K, et al. Obesity-induced gut microbial metabolite promotes liver cancer through senescence secretome. Nature 2013;499:97-101. Erratum in: Nature. 2014 Feb 20;506(7488):396. Hattori, Masahisa [corrected to Hattori, Masahira].

53. Li CY, Dempsey JL, Wang D, et al. PBDEs Altered Gut Microbiome and Bile Acid Homeostasis in Male C57BL/6 Mice. Drug Metab Dispos 2018;46:1226-40.

54. Louis P, Flint HJ. Development of a semiquantitative degenerate real-time pcr-based assay for estimation of numbers of butyrylcoenzyme A (CoA) CoA transferase genes in complex bacterial samples. Appl Environ Microbiol 2007;73:2009-12.

55. Reichardt N, Duncan SH, Young P, et al. Phylogenetic distribution of three pathways for propionate production within the human gut microbiota. ISME J 2014;8:1323-35. Erratum in: ISME J. 2014 Jun;8(6):1352. 